\title{
Simulation of spherically expanding turbulent premixed flames
}

I. Ahmed, N. Swaminathan*

Department of Engineering, Cambridge University, Cambridge, CB2 1PZ, UK.

*Corresponding author:

Department of Engineering, Cambridge University,

Trumpington Street, Cambridge, CB2 1PZ, UK.

E-mail: ns341@cam.ac.uk.

Manuscript NO.: GCST-2013-0004,

Accepted: 22 May 2013

Running Title: Simulation of spherically expanding flames 


\begin{abstract}
Statistically spherical expanding turbulent premixed flames are computed using unsteady Reynolds-Averaged Navier-Stokes (URANS) approach. Mean reaction rate is closed using strained and unstrained flamelet models and an algebraic model. The flamelets are parametrised using the scalar dissipation rate in the strained flamelet model. It is shown that this model is able to capture the measured growth rate of methane-air turbulent flame ball, which is free of thermo-diffusive instability. The spherical flames are observed to accelerate continuously. The flame brush thickness grows in time and the role of turbulent diffusion on this growth seems secondary compared to the convection due to the fluid velocity induced by the chemical reaction. The spherical flames have larger turbulent flame speed, the leading edge displacement speed $s_{t}$, compared to the planar flames for a given turbulence and thermo-chemical condition. The computational results suggest $s_{t} / s_{L}^{0} \sim \operatorname{Re}_{t}^{n}$ with $0.57 \leq n \leq 0.58$, where $\mathrm{Re}_{t}$ is the turbulence Reynolds number and $s_{L}^{0}$ is the unstrained planar laminar flame speed, for both spherical and planar flames.
\end{abstract}

Keywords: Turbulent Premixed Flames, Spherical Flames, Scalar Dissipation Rate, Turbulent Flame Speed 


\section{Introduction}

Expanding statistically spherical flame in turbulent environment is a canonically important configuration and its investigation is helpful to understand combustion in practical devices such as the spark ignited internal combustion engine, modern stratified charge engines and accidental explosions of fuel vapour cloud. Although it is a classical problem our current understanding is not fully satisfactory.

When a combustible mixture cloud is ignited at the centre, a laminar flame kernel is initiated and it develops into a turbulent spherical flame. During this evolution, the flame front is stretched due to its time varying curvature and flow straining. In addition to these effects on the flame front, the flame brush experiences stretch due to its curvature in this geometry. It is well known that the stretch rate influences the laminar flame structure and its propagation speed (Law and Sung, 2000), and these concepts are used to deduce laminar burning velocity and Markstein length scale (Bradley et al., 2009) for combustible mixtures from experiments.

Practical combustion systems involve turbulence invariably and hence turbulent spherical flames have been studied using various experimental configurations such as fan-stirred bombs involving stationary turbulence (Andrews et al., 1975; Abdel-Gayed et al., 1984; Bradley et al., 1994; Lawes et al., 2012), bombs with decaying grid turbulence (Checkel and Thomas, 1994) and wind tunnels with grid turbulence (Hainsworth, 1985; Renou et al., 2002) to address the influence of turbulence on spherical flame propagation. Beretta et al. (1983) and Hainsworth (1985) have shown that the turbulent spherical flames initially expand as the laminar flame and then it is exposed gradually to a wide range of length and time scales of turbulence resulting in flame wrinkling thereby leading to an increase in the burning velocity which is larger than the laminar value (Abdel-Gayed et al., 1987). Additional flame wrinkling can arise in 
thermo-diffusively unstable flames (of reactant mixtures with negative Markstein number). The flame wrinkling was shown to increase with pressure and for mixtures with negative Markstein numbers (Haq et al., 2002). The tendency to higher flame wrinkling resulting in faster flame propagation and high flame front curvature for mixtures with low Lewis number (thermo-diffusively unstable mixtures) is also known (Renou et al., 2000).

As a spherical flame brush expands its thickness increases, due to turbulent diffusion, with significant amount of unburnt gas inside the flame brush (Beretta et al., 1983; Abdel-Gayed et al., 1988). This poses a challenge to define the turbulent burning velocity since its definition relies on a correct choice of an associated flame radius. One way to define this radius is to equate the volume of unburnt gas inside the flame brush to that of burnt gas outside the flame brush (Bradley et al., 2003). The mass burning velocity defined using this radius is equal to the velocity of turbulent entrainment of unburnt gas into the flame brush. The flame propagation model using this entrainment concept has been developed in several past studies (Blizard and Keck, 1974; Tabaczynski et al., 1980; Groff, 1987; Bradley et al., 1994). Alternatively, flame area enhancement due to turbulence has also been considered using a vortex tube model (Ashurst et al., 1994) and an exponential growth of flame surface area (Ashurst, 1995) to study expanding spherical flames. These studies treated the flame surface to be a passive surface which is not fully satisfactory. An analogy to the theory of laminar spherical flames has also been used to study turbulent flame ball growth rate involving a turbulent Markstein number (Lipatnikov and Chomiak, 2004). These studies have helped us to develop some understanding of spherical flame propagation within the scope allowed by the assumptions used in their development.

Numerical simulations of spherical flames have been performed using Direct Numerical Simulations (DNS), Large-Eddy Simulations (LES) and Reynolds-Averaged 
Navier-Stokes (RANS) methodologies in the past. The DNS studies were initially aimed to address ignition related issues (Baum and Poinsot, 1995; Poinsot et al., 1995) using a single irreversible reaction in two-dimensional turbulence. Some of these limitations were relaxed in later DNS studies on spherical flames (Kaminski et al., 2000; Jenkins and Cant, 2002; Jenkins et al., 2006; Klein et al., 2006, 2008; Albin and D’Angelo, 2012; Thévenin et al., 2002; Thévenin, 2005; van Oijen et al., 2005) and these studies predominantly addressed flame surface density (FSD) related modelling issues. LES, in which the large energy containing scales are resolved but the flame front is modelled, has recently been used to study ignition and propagation of turbulent spherical flames (Nwagwe et al., 2000; Tabor and Weller, 2004; Fureby, 2005; Colin and Truffin, 2011; Lecocq et al., 2011). Combustion models based on sub-grid scale wrinkling factor (Nwagwe et al., 2000; Tabor and Weller, 2004; Fureby, 2005) and FSD transport equation (Colin and Truffin, 2011) have been used in conjunction with simplified chemistry to compute spherical flames. These studies showed a good comparison to the experimental data. Recently, a combustion modelling approach combining the FSD and presumed probability density function (pdf) concepts have been used (Lecocq et al., 2011) to calculate the spherical flame propagation in weak turbulence (Renou et al., 2000).

In RANS, the averaged governing equations are solved along with models for turbulent stresses and fluxes, and mean reaction rate. The RANS calculations of spherical turbulent flames of Hainsworth (1985) were done by Schmid et al. (1998) using a turbulent flame speed closure. A similar approach was also used by Lipatnikov and Chomiak (2000) to study turbulent spherical flames in various configurations. A transported joint velocity-scalar pdf approach was used by Pope and Cheng (1986) to compute the spherical flames of Hainsworth (1985) and showed a very good agreement with the measurements. 
In this work, the RANS methodology is used to study the propagation of turbulent premixed spherical and planar flames. The reaction rate is modelled using the strained flamelet model (Kolla and Swaminathan, 2010), where the flamelets are parametrised using scalar dissipation rate of a progress variable. The progress variable is defined as $c=\left(T-T_{u}\right) /\left(T_{b}-T_{u}\right)$, where $T_{b}$ and $T_{u}$ denote the burnt and unburnt mixture temperature respectively. The Favre-averaged scalar dissipation rate is defined as $\tilde{\epsilon}_{c}=$ $\overline{\rho \alpha_{c}\left(\nabla c^{\prime \prime} \cdot \nabla c^{\prime \prime}\right)} / \bar{\rho}$, where $c^{\prime \prime}$ is the Favre fluctuation of $c$ with the molecular diffusivity $\alpha_{c}$. More detail of this modelling approach is described in the next section. The main objectives of this work are twofold: first to validate the applicability of the strained flamelets approach for turbulent spherical flames and to compare its performance with other flamelet based combustion models; second to contrast the flame propagation mechanisms in spherical and planar cases and to elucidate the underlying physics.

This paper is organised as follows. The governing equations, reaction rate model and numerical setup are discussed in the next section. The experimental test case for validation and various computational cases considered are described in Section 3. The simulation results are presented and discussed in Section 4. The main conclusions of this study are summarised in the last section.

\section{Numerical Setup}

\subsection{Governing equations and modelling}

The unsteady RANS (URANS) approach is used to simulate spherical turbulent explosions in premixed methane-air mixtures. These flames are assumed to be spherically symmetric resulting in considerable simplification as it allows us to retain only the radial terms of the governing equations written in $(r, \theta, \phi)$ coordinates. 
The Favre-averaged equations for the mass and radial momentum conservation are

$$
\begin{aligned}
& \frac{\partial \bar{\rho}}{\partial t}+\frac{1}{r^{2}} \frac{\partial r^{2} \bar{\rho} \tilde{u}_{r}}{\partial r}=0, \\
& \frac{\partial \bar{\rho} \tilde{u}_{r}}{\partial t}+\frac{1}{r^{2}} \frac{\partial}{\partial r}\left[r^{2} \bar{\rho} \tilde{u}_{r}^{2}\right]=-\frac{\partial \bar{p}}{\partial r}+\frac{1}{r^{2}} \frac{\partial}{\partial r}\left[r^{2}\left(\bar{\tau}_{r r}-\overline{\rho u_{r}^{\prime \prime 2}}\right)\right] \\
&- \frac{\left(\bar{\tau}_{\theta \theta}-\overline{\rho u_{\theta}^{\prime \prime 2}}+\bar{\tau}_{\phi \phi}-\overline{\rho u_{\phi}^{\prime \prime 2}}\right)}{r},
\end{aligned}
$$

where $\tau_{r r}, \tau_{\theta \theta}$ and $\tau_{\phi \phi}$ denote the normal components of the viscous stress tensor in the respective directions. The centrifugal forces per unit volume arising from the Reynolds stresses in $\theta$ and $\phi$ directions are $\overline{\rho u_{\theta}^{\prime \prime 2}} / r$ and $\overline{\rho u_{\phi}^{\prime \prime 2}} / r$ respectively, which do not vanish even in the spherically symmetric case. Thus, they must be retained because their contributions are significant in the earlier period of flame development (small $r$ ).

The uncertainties related to turbulence modelling is minimised by using the $k-\varepsilon$ equations given by

$$
\begin{aligned}
\frac{\partial \bar{\rho} \tilde{k}}{\partial t}+\frac{1}{r^{2}} \frac{\partial}{\partial r}\left[r^{2} \bar{\rho} \tilde{u}_{r} \tilde{k}\right] & =\frac{1}{r^{2}} \frac{\partial}{\partial r}\left\{r^{2}\left[\left(\mu+\frac{\mu_{t}}{\mathrm{Sc}_{k}}\right) \frac{\partial \tilde{k}}{\partial r}\right]\right\}-\overline{\rho u_{r}^{\prime \prime 2}}\left(\frac{\partial \tilde{u}_{r}}{\partial r}\right) \\
& -\left(\overline{\rho u_{\theta}^{\prime \prime 2}}+{\overline{\rho u_{\phi}^{\prime \prime}}}^{2}\right) \frac{\tilde{u}_{r}}{r}-\overline{u_{r}^{\prime \prime}} \frac{\partial \bar{p}}{\partial r}+\overline{p^{\prime} \nabla \cdot \boldsymbol{u}^{\prime \prime}}-\bar{\rho} \tilde{\varepsilon}, \\
\frac{\partial \bar{\rho} \tilde{\varepsilon}}{\partial t}+\frac{1}{r^{2}} \frac{\partial}{\partial r}\left[r^{2}\left(\bar{\rho} \tilde{u}_{r} \tilde{\varepsilon}\right)\right] & =\frac{1}{r^{2}} \frac{\partial}{\partial r}\left\{r^{2}\left[\left(\mu+\frac{\mu_{t}}{\mathrm{Sc}_{\varepsilon}}\right) \frac{\partial \tilde{\varepsilon}}{\partial r}\right]\right\}-C_{\varepsilon 1} \frac{\tilde{\varepsilon}}{\tilde{k}}\left[\overline{\rho u_{r}^{\prime \prime 2}}\left(\frac{\partial \tilde{u}_{r}}{\partial r}\right)\right. \\
& \left.+\left(\overline{\rho u_{\theta}^{\prime \prime 2}}+{\overline{\rho u_{\phi}^{\prime \prime}}}^{2}\right) \frac{\tilde{u}_{r}}{r}-\overline{u_{r}^{\prime \prime}} \frac{\partial \bar{p}}{\partial r}\right]-C_{\varepsilon 2} \bar{\rho} \frac{\tilde{\varepsilon}^{2}}{\tilde{k}},
\end{aligned}
$$

where $\mu$ and $\mu_{t}$ represent the molecular and eddy viscosities respectively. The model constants are $C_{\epsilon 1}=1.44, C_{\epsilon 2}=1.92$ and $\mathrm{Sc}_{k}=\mathrm{Sc}_{\epsilon}=1$. The second and third terms appearing on the RHS of Eq. (3) represent the production of $\tilde{k}$ by the gradients of 
mean velocity. The next two terms respectively represent the effects of mean pressure gradient and pressure-dilatation. The dissipation of $\tilde{k}$ is represented by the last term of Eq. (3).

The Reynolds stresses are modelled using the eddy-viscosity hypothesis as

$$
\begin{aligned}
& \overline{\rho u_{r}^{\prime \prime 2}}=-2 \mu_{t} \frac{\partial \tilde{u}_{r}}{\partial r}+\frac{2}{3} \mu_{t}\left[\frac{1}{r^{2}} \frac{\partial}{\partial r}\left(r^{2} \tilde{u}_{r}\right)\right]+\frac{2}{3} \bar{\rho} \tilde{k}, \\
& \overline{\rho u_{\theta}^{\prime \prime 2}}=\overline{\rho u_{\phi}^{\prime \prime 2}}=-2 \mu_{t} \frac{\tilde{u}_{r}}{r}+\frac{2}{3} \mu_{t}\left[\frac{1}{r^{2}} \frac{\partial}{\partial r}\left(r^{2} \tilde{u}_{r}\right)\right]+\frac{2}{3} \bar{\rho} \tilde{k} .
\end{aligned}
$$

If one uses an anisotropic turbulence model then $\overline{\rho u_{\theta}^{\prime \prime 2}}$ and $\overline{\rho u_{\phi}^{\prime 2}}$ will be different. The pressure work and pressure-dilatation terms are often neglected or combined with the diffusive term in reacting flow simulations and these are modelled explicitly in this study. The pressure-dilatation is modelled as $\overline{p^{\prime} \boldsymbol{\nabla} \cdot \boldsymbol{u}^{\prime \prime}}=0.5 \tilde{c}\left(\tau s_{L}^{0}\right)^{2} \dot{\dot{\omega}}$ (Zhang and Rutland, 1995). The $\overline{u^{\prime \prime}}$ in the pressure work term is modelled (Libby, 1985) as $\overline{\boldsymbol{u}^{\prime \prime}}=\widetilde{\boldsymbol{u}^{\prime \prime} \boldsymbol{c}^{\prime \prime}} \tau /(1+\tau \tilde{\boldsymbol{c}})$, where the turbulent scalar flux $\widetilde{\boldsymbol{u}^{\prime \prime} \boldsymbol{c}^{\prime \prime}}$ is modelled using the classical gradient transport. It is well known that this scalar flux can be counter gradient in premixed flames, which can be included in simulations using second order closures. However, the gradient model is used in this study for the sake of simplicity and its validity can be evaluated from the experimental comparisons to be shown later in section 4.1. Furthermore, it is well known that gradient flux dominates when $u^{\prime} / s_{L}^{0} \geq 4$ (Veynante et al., 1997; Swaminathan et al., 1997; Kalt et al., 1998; Frank et al., 1999) and the flames considered in this study have $u^{\prime} / s_{L}^{0}>4$ as one shall see later in section 3.2. Although it is ideal to include the pressure-dilatation effect in both $\tilde{k}$ and $\tilde{\varepsilon}$ equations it is included only in $\tilde{k}$ equation following many previous studies (Bray et al., 1985; Jones, 1994; Kolla and Swaminathan, 2010). The effects of these terms may be small for open flames (Swaminathan and Bray, 2011).

The mean thermo-chemical state of the mixture is obtained using the progress vari- 
able equation:

$$
\frac{\partial \bar{\rho} \tilde{c}}{\partial t}+\frac{1}{r^{2}} \frac{\partial}{\partial r}\left(r^{2} \bar{\rho} \tilde{u}_{r} \tilde{c}\right)=\frac{1}{r^{2}} \frac{\partial}{\partial r}\left[r^{2}\left(\overline{\rho \alpha}_{c} \frac{\partial \tilde{c}}{\partial r}-\overline{\rho u_{r}^{\prime \prime} c^{\prime \prime}}\right)\right]+\overline{\dot{\omega}}
$$

where $\overline{\dot{\omega}}$ is the mean rate of production of $\tilde{c}$ per unit volume, which is modelled using the strained flamelet model explained in section 2.2. This model requires the progress variable variance, $\widetilde{c^{\prime \prime 2}}$, and thus its transport equation

$$
\begin{aligned}
\frac{\partial \bar{\rho} \widetilde{c^{\prime \prime 2}}}{\partial t}+\frac{1}{r^{2}} \frac{\partial}{\partial r}\left(r^{2} \bar{\rho} \tilde{u}_{r} \widetilde{c^{\prime \prime 2}}\right) & =\frac{1}{r^{2}} \frac{\partial}{\partial r}\left\{r^{2}\left[\left(\mu+\frac{\mu_{t}}{\mathrm{Sc}_{c}}\right) \frac{\partial \widetilde{c^{\prime \prime 2}}}{\partial r}\right]\right\} \\
& -2 \overline{\rho u_{r}^{\prime \prime} c^{\prime \prime}} \frac{\partial \tilde{c}}{\partial r}-2 \bar{\rho} \tilde{\epsilon}_{c}+2 \overline{\dot{\omega}^{\prime \prime} c^{\prime \prime}}
\end{aligned}
$$

is also included in the simulation. Its source term is obtained using $\overline{\dot{\omega}^{\prime \prime} c^{\prime \prime}}=\overline{\dot{\omega} c}-\overline{\dot{\omega}} \tilde{c}$. The dissipation rate, $\tilde{\epsilon}_{c}$, is closed using the model described in the next subsection. The mean density is calculated using the equation of state, $\bar{\rho}=\rho_{u} /(1+\tau \tilde{c})$.

\subsection{Reaction rate model}

The mean reaction rate, $\overline{\dot{\omega}}$, is modelled using the strained flamelet model, which is described briefly here and elaborate detail can be found in Kolla and Swaminathan (2010). The flamelets, which are freely propagating laminar flame and those established in opposing flows of reactant and product, are parametrised using $\tilde{\epsilon}_{c}$. The mean reaction rate is given by

$$
\overline{\dot{\omega}}=\int_{0}^{1}\left[\int_{N_{1}}^{N_{2}} \dot{\omega}(\zeta, \psi) P(\psi \mid \zeta) \mathrm{d} \psi\right] P(\zeta) \mathrm{d} \zeta
$$

where $\zeta$ and $\psi$ are the sample space variables for $c$ and the instantaneous scalar dissipation rate, $N$, respectively. The flamelet reaction rate, $\dot{\omega}(\zeta, \psi)$, and the integration 
limits $N_{1}$ and $N_{2}$ are obtained using results of fully burning and almost extinguished flamelets. An arbitrarily complex chemical kinetic mechanism can be used in the flamelet calculation.

The presumed shapes for the pdfs, $P(\zeta)$ and $P(\psi \mid \zeta)$, are specified using the $\beta$ and lognormal functions respectively. The $\beta$ function requires $\tilde{c}$ and $\widetilde{c^{\prime \prime 2}}$, and the lognormal form for $P(\psi \mid \zeta)$ requires the conditional mean and variance of the natural logarithm of the conditional scalar dissipation rate, i.e., $\ln (N \mid \zeta)$. These dissipation related quantities are obtained following the method described by Kolla and Swaminathan (2010). For given values of $\tilde{c}, \widetilde{c^{\prime \prime 2}}$ and $\tilde{\epsilon}_{c}$, one can build a table for $\overline{\dot{\omega}}$ and other required quantities using the laminar flame solutions as per Eq. (9). This look-up table is referred during turbulent flame simulations to obtain $\overline{\dot{\omega}}$ and $\overline{\dot{\omega} c}$ required to close Eqs. (7) and (8).

The mean and variance required for the look-up are obtained from the respective transport equations. Many models available for $\tilde{\epsilon}_{c}$ are discussed by Chakraborty et al. (2011) and the model of Kolla et al. (2009) is used in this study because it is simple and satisfies the realisability condition $\left(\tilde{\epsilon}_{c} \geq 0\right)$. This model written as

$$
\tilde{\epsilon}_{c} \simeq \frac{1}{\beta^{\prime}}\left[\left(2 K_{c}^{*}-\tau C_{4}\right) \frac{s_{L}^{0}}{\delta_{L}^{0}}+C_{3} \frac{\tilde{\varepsilon}}{\tilde{k}}\right] \widetilde{c^{\prime \prime 2}}
$$

was obtained by balancing the leading order terms of a transport equation for $\tilde{\epsilon}_{c}$ derived by Swaminathan and Bray (2005). The various model parameters are: $\beta^{\prime}=6.7, K_{c}^{*}=$ $0.85 \tau$ (for hydrocarbon-air mixtures), $C_{3}=1.5 \sqrt{\mathrm{Ka}} /(1+\sqrt{\mathrm{Ka}})$ and $C_{4}=1.1 /(1+$ $\mathrm{Ka})^{0.4}$. These parameters are specified to satisfy certain physical aspects of turbulenceflame interaction (Kolla et al., 2009; Kolla and Swaminathan, 2011) and so they cannot be changed arbitrarily. The Karlovitz number is defined as $\mathrm{Ka} \equiv\left(u^{\prime} / s_{L}^{0}\right)^{3 / 2}(\delta / \Lambda)^{1 / 2}$, where $u^{\prime}=\sqrt{2 \tilde{k} / 3}, \Lambda=u^{\prime 3} / \tilde{\varepsilon}$ and $\delta_{L}^{0}=\delta\left[2(1+\tau)^{0.7}\right]$.

The dissipation rate model in Eq. (10) includes the effects of curvature induced 
stretch on flamelets and various important effects of turbulence and its interaction with chemical reaction and molecular diffusion. The parameter $\beta^{\prime}$ represent the flamelet curvature induced effects. However, a spherical flame brush also experiences stretch due to its mean curvature, which is absent in a planar case. This particular effect is not included in Eq. (10) and thus an additional correction can be included based on the analysis of Chakraborty et al. (2010). This revised model written as

$$
\tilde{\boldsymbol{\epsilon}}_{c} \simeq \frac{1}{\beta^{\prime}}\left\{\left[2 K_{c}^{*}-\tau C_{4}\left(1-\frac{\alpha_{u}}{s_{L}^{0}} \nabla \cdot \boldsymbol{n}\right)\right] \frac{s_{L}^{0}}{\delta_{L}^{0}}+C_{3} \frac{\tilde{\varepsilon}}{\tilde{k}}\right\} \widetilde{c^{\prime \prime 2}}
$$

is obtained through a leading order balance analysis, similar to Kolla et al. (2009), using the models proposed by Chakraborty et al. (2010). The normal vector in Eq. (11) is defined as $\boldsymbol{n}=-\nabla \tilde{c} /|\nabla \tilde{c}|$. The major difference between the models in Eqs. (10) and (11) is the contribution of flame brush curvature $\boldsymbol{\nabla} \cdot \boldsymbol{n}$. Note that the revised model in Eq. (11) is unconditionally realisable for explosion but the realisability condition imposes a minimum radius for implosion. Both models in Eqs. (10) and (11) are used in this study to understand the extent of influence of $\boldsymbol{\nabla} \cdot \boldsymbol{n}$.

The strained flamelet model is also compared to two other models. One of them is the algebraic model (Bray, 1979):

$$
\overline{\dot{\omega}}=\frac{2}{2 C_{m}-1} \bar{\rho} \tilde{\epsilon}_{c} \quad \text { and } \quad \overline{\dot{\omega}^{\prime \prime} c^{\prime \prime}}=\left(C_{m}-\tilde{c}\right) \overline{\dot{\omega}}
$$

with $C_{m} \approx 0.7$ (Swaminathan and Bray, 2005) and either of Eqs. (10) or (11) can be used for $\tilde{\epsilon}_{c}$ in Eq. (12) and this equation does not involve complex chemical kinetics. The other one is unstrained flamelet model:

$$
\overline{\dot{\omega}}=\int_{0}^{1} \dot{\omega}_{o}(\zeta) P(\zeta) \mathrm{d} \zeta \quad \text { and } \quad \overline{\dot{\omega}^{\prime \prime} c^{\prime \prime}}=\int_{0}^{1} \zeta \dot{\omega}_{0}(\zeta) P(\zeta) \mathrm{d} \zeta-\overline{\dot{\omega}} \tilde{c}
$$


where $\dot{\omega}_{o}$ is the unstrained flamelet reaction rate obtained from the planar unstrained laminar flame calculation involving complex chemistry. Unlike the strained flamelet model, the look-up table for this model is two-dimensional involving $\tilde{c}$ and $\widetilde{c^{\prime \prime 2}}$.

\subsection{Computational approach and parameters}

The governing equations along with the various models discussed in the previous two subsections are solved using finite-volume methodology. The power law scheme was used to discretise spatial gradients and an implicit first order backward Euler method is used for time stepping. The pressure-velocity coupling is through the SIMPLER approach of Patankar (1980).

The flamelets required for the look-up table construction are calculated using PREMIX (Kee et al., 1985) and OPPDIF (Lutz et al., 1997) codes. The GRI-Mech 3.0 (Smith et al., 2000) is used for combustion kinetics of methane-air mixture. The look-up table has $\overline{\dot{\omega}}$ as a function of $\tilde{c}, \widetilde{c^{\prime \prime 2}}$ and $\tilde{\epsilon}_{c}$ and a tri-linear interpolation is used to obtain $\overline{\dot{\omega}}$ for the computed values of $\tilde{c}, \widetilde{c^{\prime \prime 2}}$ and $\tilde{\epsilon}_{c}$ at a given grid point during the URANS calculation.

The computational domain length varies from 0.2 to $1 \mathrm{~m}$, depending on the ratio of turbulence integral length scale $\Lambda$ to the Zeldovich thickness $\delta$, so that the simulated statistically planar and spherical flames remain within the domain for the simulation period. The number of grid points are chosen so that there are at least 10 points inside $\min \left(\Lambda, \delta_{t}\right)$, where $\delta_{t} \equiv 1 /|\partial \tilde{c} / \partial r|_{\max }$ is the turbulent flame brush thickness, for a uniform grid spacing. The size of time-step is chosen to be $0.1 \mu \mathrm{s}$, which ensures the resolution of reaction, diffusion and convection time scales and satisfies the numerical stability conditions for the chosen grid, turbulence and thermo-chemical conditions.

The values of $\tilde{k}$ and $\tilde{\varepsilon}$ chosen for the unburnt mixture are specified over the entire computational domain initially. The initial spatial variation of $\tilde{c}$ having 0 in the unburnt 
and 1 in the burnt mixtures is chosen after few tests to minimise the initial transients for the given turbulence and thermo-chemical conditions in order to save computational time required to attain a "steady propagation" state. The initial $\tilde{u}, \bar{\rho}$ and $\bar{p}$ are specified to be consistent with the initial $\tilde{c}$ variation. It is straightforward to specify the boundary conditions for the planar flames as has been done in many earlier studies (Corvellec et al., 1999, 2000; Swaminathan and Bray, 2005; Kolla and Swaminathan, 2010). The following boundary conditions apply for a flame propagating radially outward in an unconfined domain:

$$
\tilde{u}_{r}(0, t)=\left.\frac{\partial \tilde{c}}{\partial r}\right|_{(0, t)}=\left.\frac{\partial \tilde{k}}{\partial r}\right|_{(0, t)}=\left.\frac{\partial \tilde{\varepsilon}}{\partial r}\right|_{(0, t)}=\left.\frac{\partial \widetilde{c^{\prime \prime 2}}}{\partial r}\right|_{(0, t)}=0
$$

For the unburnt mixture $\left(r=r_{1}\right)$

$$
\left.\frac{\partial \tilde{u}_{r}}{\partial r}\right|_{\left(r_{1}, t\right)}=\left.\frac{\partial \tilde{k}}{\partial r}\right|_{\left(r_{1}, t\right)}=\left.\frac{\partial \tilde{\varepsilon}}{\partial r}\right|_{\left(r_{1}, t\right)}=0, \quad \widetilde{c^{\prime \prime 2}}\left(r_{1}, t\right)=\tilde{c}\left(r_{1}, t\right)=0, \quad \bar{p}\left(r_{1}, t\right)=p_{\infty}
$$

The values of turbulent kinetic energy, $\tilde{k}$, and its dissipation rate, $\tilde{\varepsilon}$, obtained using the chosen value of $u^{\prime}$ and $\Lambda$ are used to specify their initial conditions.

\section{Test Flames}

The numerical models described in the previous section are used to study the influence of turbulence and thermo-chemical conditions on the evolution of expanding spherical flames. The simulation results will also be used to elucidate the difference in the propagation of planar and spherical flames. Before discussing the conditions of these flames considered here, an experimental case used to validate the numerical models is described briefly. 


\subsection{Validation case}

The spherical flames established in wind tunnel turbulence by Hainsworth (1985) are considered for model validation purpose. This wind tunnel turbulence generated using perforated plates was homogeneous and isotropic at the spark location, and the methane-air mixture having equivalence ratios of $\Phi=1.1$ and 0.8 were considered. For the reasons to be discussed in subsection 4.1, $\Phi=1.1$ mixture is considered for this study and its thermo-chemical characteristics along with the experimental conditions at ignition are given in Table 1 . The flame was ignited using a spark downstream of the perforated plate and it was convected downstream by the mean flow as it evolves in an approximately spherical shape. Temporal changes of position and radius of this flame were recorded using high speed schlieren movies and it has been suggested that this flame is representative of combustion in spark-ignition engines (Pope, 1987). This flame was also considered in earlier computational studies (Pope and Cheng, 1986; Schmid et al., 1998; Lipatnikov and Chomiak, 2000).

Table 1: Experimental conditions for $\Phi=1.1$ flame of Hainsworth (1985)

\begin{tabular}{rl|rl}
\hline$s_{L}^{0}$ & $=0.43 \mathrm{~m} / \mathrm{s}$ & $u^{\prime}$ & $=1.93 \mathrm{~m} / \mathrm{s}$ \\
$\delta_{L}^{0}$ & $=0.0408 \mathrm{~cm}$ & $\Lambda$ & $=0.838 \mathrm{~cm}$ \\
$\delta$ & $=0.00565 \mathrm{~cm}$ & $p_{0}$ & $=0.1 \mathrm{MPa}$ \\
$r_{f, 0}$ & $=0.15 \mathrm{~cm}$ & $T_{0}$ & $=298 \mathrm{~K}$ \\
$\tau$ & $=5.25$ &
\end{tabular}

\subsection{Flames for further analysis}

Spherical flames propagating outwardly in nearly homogeneous isotropic turbulence field in an unconfined space are considered. The boundary conditions discussed earlier in subsection 2.3 describe this problem. The influences of combustion on turbulence are also included in the simulation by solving the $\tilde{k}-\tilde{\varepsilon}$ equation. A stoichiomet- 
ric methane-air mixture at $298 \mathrm{~K}$ and atmospheric pressure is considered for these flames. Since this mixture has unity Lewis number, the influence of turbulence on the flame propagation can be studied without the added complexity of differential diffusion, which could amplify the stretch induced effects. Furthermore, this mixture was considered in an earlier study addressing the turbulence effects on the propagation of statistically planar flames (Kolla and Swaminathan, 2010). Thus, the behaviour of spherical flames can be compared directly to the planar flame results to understand the geometry effects. The thermo-chemical characteristics of this mixture are, $s_{L}^{0}=0.4 \mathrm{~m} / \mathrm{s}, \delta_{L}^{0}=0.41 \mathrm{~mm}, \tau=6.48$ and $\delta=0.047 \mathrm{~mm}$.

The turbulent combustion conditions of 8 flames simulated in this study are shown in Figure 1. Two different values for the stretch factor, as defined by Abdel-Gayed et al. (1987), $K=0.157\left(u^{\prime} / s_{L}^{0}\right)^{2} \operatorname{Re}_{t}^{-0.5}=0.157$ and 1 are considered. The turbulence Reynolds number is defined as $\operatorname{Re}_{t}=u^{\prime} \Lambda / v$. The flames with the smaller stretch factor value have the Karlovitz number, $\mathrm{Ka}=\left(u^{\prime} / s_{L}^{0}\right)^{2} \operatorname{Re}_{t}^{-0.5}$, of unity and they are located at the upper limit of the corrugated flamelets regime. The other case with larger $K$ value is in the thin reaction zones regime as in Figure 1. These particular values for $K$ are chosen so that the combustion conditions remain the same for the current spherical and planar flames of Kolla and Swaminathan (2010). For the three flames with $K=0.15$, the values of $u^{\prime} / s_{L}^{0}$ are 5,6 and 8, and these values are 12,16, 18, and 20 for the other cases with $K=1$. It is also to be noted that the experimental flame of Hainsworth (1985) is in the corrugated flamelets regime for the conditions noted in table 1.

\section{Results and discussion}

The computational results of these spherically expanding flames under a wide range of turbulence conditions are analysed in this section. Validation of the computational 
models are discussed first. Then, the influence of turbulence on the propagation of spherical flames are explored using the seven flames and they are compared with the corresponding planar flames to understand the geometry effects.

\subsection{Model Validation}

Figure $2 \mathrm{a}$ shows the temporal variation of the flame brush radius measured using high speed schlieren techniques for $\Phi=1.1$ mixture in two sets of experiments (Hainsworth, 1985). Since the schlieren images show the burnt side and marks the regions with strong density gradients, the flame radius reported in the experiment is taken to be the leading edge of the flame brush (Bradley et al., 2000, 2011). For comparison purposes, the location at which $\tilde{c}=0.05$ is taken to be the leading edge in the simulated flames. As one expects, this radius grows with time as in Figure 2a, where the radius is normalised using its initial value, $r_{f, 0}$ in table 1 , and the time is normalised using the laminar flame time or chemical time, $t_{c}=\delta_{L}^{0} / s_{L}^{0}$, (see table 1$)$. The bottom two curves, marked for laminar flames, represent the evolution of the initial flame kernel if it evolves as a laminar spherical flame. This laminar evolution can be computed simply by considering the mass conservation, $\mathrm{d} m / \mathrm{d} t=\rho_{u} s_{L} A$, for the burnt gas mass, $m$, inside the kernel having a surface area of $A$. This simplifies to $\mathrm{d} r_{f} / \mathrm{d} t=\left(\rho_{u} / \rho_{b}\right) s_{L}$ for a spherical kernel. If one takes $s_{L}=s_{L}^{0}$ ignoring the stretch effects on the laminar flame propagation then $r_{f}$ grows linearly with $t$ and this line passes through the experimental data for $t^{+} \leq 2$, suggesting that the initial evolution is laminar and it may be uninfluenced by the stretch effects induced by flow straining and curvature. This is supported by the result shown for stretched laminar flame in Figure 2a (the bottom most curve). The stretch effects are included in the above mass conservation equation by expressing $s_{L}=s_{L}^{0}-\kappa \mathcal{L}$, where $\kappa=2\left(\mathrm{~d} \ln r_{f} / \mathrm{d} t\right)$ is the stretch rate and $\mathcal{L}$ is the Markstein length scale for the chosen mixture. This length scale is computed as 
$0.89 \mathrm{~mm}$ using Eq. (2.109) of Poinsot and Veynante (2005) which is very close to the value reported by Bradley et al. (1996). The comparison of unstretched and stretched laminar flame results to the experimental data suggests the following. It was clearly noted by Hainsworth (1985) that the mixture of $\Phi=1.1$ is thermo-diffusively stable and there is no cell formation on the flame surface. Hence, the increase in the burning rate is purely due to turbulence. As the initial laminar flame grows, it is exposed to progressively wider range of scales which would increase the surface area through flame wrinkling which results in increased burning rate as has been noted in earlier studies (Beretta et al., 1983; Hainsworth, 1985). It is possible that this increase is compensated by the stretch induced negative effect, resulting in a growth rate similar to that of freely propagating spherical laminar flame for about $t^{+} \leq 2$. Beyond this time, the effects of flame wrinkling produced by the turbulence overwhelms the stretch effect producing a smooth departure from the freely propagating spherical laminar flame as seen in Figure 2a.

When the flame kernel is much smaller than the turbulence integral length scale, it will simply be convected by the large scale eddy and the flame-turbulence interaction is limited to very small part of a wide spectrum of turbulence scales and thus the RANS combustion modelling may not hold. This leads to some ambiguity in using the turbulent combustion modelling to simulate the transition from laminar to turbulent growth using the URANS approach. This has been recognised by Pope (1987) and so, a joint velocity-scalar pdf approach was chosen by Pope and Cheng (1986) to simulate this experimental flame from $t^{+}=0$, showing a good comparison with measurements over the whole period of the experiment. Thus, the experimentally measured flame radius at $t^{+} \approx 2.5$ obeying this condition is chosen as the initial flame radius for the simulation. This flame radius departs from the laminar result by about $5 \%$ as shown in Figure $2 \mathrm{a}$. To be consistent with the conditions of turbulence at $t^{+} \approx 2.5$ in the experiment, a cold 
flow simulation starting with the conditions in table 1 was run up to $t^{+} \approx 2.5$ (corresponding to about $2.4 \mathrm{~ms}$ ) and the data from this cold flow simulation were used as initial conditions for the turbulent flame calculations reported in Figure 2a. Thus, the turbulent flame results start from $t^{+} \approx 2.5$ in this figure.

The URANS approach along with the $k-\varepsilon$ model was also used in earlier studies employing an empirical mean reaction rate model based on turbulent flame speed closure (Schmid et al., 1998) and a time dependent mean reaction rate closure with a laminar-like source (Lipatnikov and Chomiak, 2000). The later study also excluded momentum equation in the analysis.

The difficulties noted above due to the relative size of the flame and turbulence integral length scale and further reasonings given below have limited us to choose only $\Phi=1.1$ flame from the experiments of Hainsworth (1985) for this study. Also, the lean methane-air mixture is thermo-diffusively unstable (weakly) and combustion modelling must take this effect duly. It is unclear at this time how to include these effects into RANS combustion modelling. As shown in Figure 1, the conditions of the experimental flames are in the corrugated flamelets regime and whether one can ignore the thermo-diffusive instabilities, however weak they may be, and their influence on flame propagation is an open question.

The turbulent flame results are shown for three different combustion models in Figure 2a. The algebraic model in Eq. (12) over predicts the flame growth as one would expect because this model assumes fast chemistry resulting in faster burning. The unstrained flamelet model in Eq. (13) includes finite rate chemistry effects but assumes the flame front to be a freely propagating laminar flame and thus excludes the local stretch effects on the flame front. Thus, the flame growth rate is over predicted by this model also, but the level of over prediction is reduced by a small amount from the algebraic model case. The values of $r_{f}$ computed using the strained flamelet model 
given by Eq. (9) agrees well with the measured values as shown in Figure 2a for the following reason. In premixed flames, the local balance among reaction, diffusion and fluid dynamic effects determines the local scalar gradient magnitude which is directly related to the scalar dissipation rate. The stretch effects from turbulence are due to straining and curvature and both of these will directly influence the scalar gradient. Thus, using the scalar gradient to parametrise the flamelets seems prudent for spherical flames also as it has been shown earlier for planar flames (Kolla and Swaminathan, 2010). The relative behaviour of the three combustion models shown here for the statistically spherical flame is similar to the observation of Kolla and Swaminathan (2010) for statistically planar flames. Also, the use of equation (11) to include the curvature of the flame brush shows negligible effect on the growth of the flame as in Figure 2a and for this reason we shall use Eq. (10) for further analyses of spherical flames to be discussed below, unless mentioned otherwise.

There is some uncertainty in choosing the initial flame radius for the computations as noted earlier. Thus, the variation of normalised propagation speed, $\left(\mathrm{d} r_{f} / \mathrm{d} t\right) / s_{L}^{0}$, with the normalised radius is also shown in Figure 2b. The computational results are about $12 \%$ larger than the values derived from the experimental results and this level of difference is acceptable. A best fit cubic curve for the two set of experimental data for $t>2.4 \mathrm{~ms}$ given in Figure $2 \mathrm{a}$ is used to calculate $\mathrm{d} r_{f} / \mathrm{d} t$ for the experimental result.

\subsection{Spherical and planar flames comparison}

The flame geometry effect on the propagation and consumption speeds of turbulent premixed flames is investigated in this section using the results of spherical and planar flames simulated in this study. The planar flame results computed in this study were observed to be very close to those reported by Kolla and Swaminathan (2010). All the 
flames investigated in this section are simulated for a period of about $8 \mathrm{~ms}$. Typical time evolution of these two, planar and spherical, flames is shown in Figure 3 by plotting the spatial variation of $\tilde{c}$ at various times. The spatial position, $x^{\prime}$, shown in this figure is a Galilean transformed, $x^{\prime}=x-\widetilde{u}_{b} t$, because the burnt side velocity, $\widetilde{u}_{b}$, is different in the planar and spherical cases. This allows a direct comparison between these two flames. The burnt side velocity is zero in the spherical case and it is negative in the planar case.

The initial variation is shown by dashed lines and the profiles are shown for a period of $8 \mathrm{~ms}\left(t^{+} \approx 7.8\right)$ at an interval of $2 \mathrm{~ms}$. These flames have $u^{\prime} / s_{L}^{0}=6$ and $K=$ 0.15 , and the same thermochemical parameters since they are stoichiometric $\mathrm{CH}_{4}$-air mixture. These flames propagate from left to right in Figure 3 and they are computed in the cartesian and spherical coordinate systems respectively. This flame pair is used to demonstrate the flame geometry effects since the relative behaviours shown and discussed in this section hold for other cases considered, unless noted otherwise.

Figure 3 shows that the numerical resolution used is excellent. This figure shows the planar flame reaches a nearly steady propagation speed after some initial transients but the spherical flame does not seem to suggest a steady value for its propagation speed (shown in Figure $3 b$ by increasing gap between the consecutive iso-contour profiles). As the spherical flame grows outwardly the leading surface area increases resulting in increased burning rate, which can be seen clearly by plotting the temporal variation of the propagation speed of an iso-value, $\tilde{c}=c_{1}$. This speed is extracted from the computed time variation of the spatial position $x\left(c_{1}\right)$ or $r\left(c_{1}\right)$ through

$$
\frac{d x\left(c_{1}\right)}{d t} \cdot \boldsymbol{n}=\tilde{u} \cdot \boldsymbol{n}+s_{d}
$$

where $\tilde{u}$ is the fluid velocity and $s_{d}$ is the displacement speed of the iso-level in its 
normal, $\boldsymbol{n}$, direction due to its relative movement created by combined effects of mean reaction rate, turbulent flux and molecular diffusion. The effect of molecular diffusion can be neglected in high Reynolds number turbulent flows and the displacement speed can then be written as

$$
s_{d}=\underbrace{\frac{1}{r^{2}} \frac{\partial}{\partial r}\left[r^{2}\left(\frac{\mu_{t}}{\operatorname{Sc}_{c}} \frac{\partial \tilde{c}}{\partial r}\right)\right] /\left(\bar{\rho}\left|\frac{\partial \tilde{c}}{\partial r}\right|\right)}_{s_{d}^{T}}+\underbrace{\overline{\dot{\omega}} /\left(\bar{\rho}\left|\frac{\partial \tilde{c}}{\partial r}\right|\right)}_{s_{d}^{r}},
$$

using the mean progress variable equation, Eq. (7). It is understood that all the quantities on the right hand side of Eq. (17) must be evaluated at $\tilde{c}=c_{1}$. A corresponding equation can also be written in the Cartesian system. The displacement speed of the leading edge, $s_{d}(\tilde{c}=0.05)$ is referred as the turbulent flame speed in the latter part of this section. The equality in Eq. (16) is verified using the computational results since the three terms can be evaluated individually.

Figure 4 shows the temporal variation of the propagation speed with respect to the burnt mixture computed from $\mathrm{d} x^{\prime} / \mathrm{d} t$ for the iso-levels. The results are shown for three iso-levels, $\tilde{c}=0.05,0.5$ and 0.8 . The cases with low and high turbulence level are shown respectively in Figure $4 \mathrm{a}$ and $4 \mathrm{~b}$. This propagation speed is normalised using the unstrained planar laminar flame speed and the time is normalised using the respective integral time scale of the turbulence in the reactants, $t_{e}$. This normalised time is related to $t^{+}$through $t^{*}=t^{+}\left(t_{c} / t_{e}\right)$. After going through some initial transients for $t^{+} \sim 2$ to 2.5 all the iso-levels converge to a nearly constant propagation speed which depends on the value of $u^{\prime} / s_{L}^{0}$ for the planar flames and a small decrease with $t^{*}$ suggests the persistence of the initial transients. On the other hand, this propagation speed increases with $t^{*}$ and different iso-levels are travelling at different speeds in spherical flames. The larger value seen in the early period for the planar flames is because of high $\widetilde{u}_{b}$. The continuous growth in the spherical cases is because of the continuous increase in the 
burning surface area. Further discussion on this point is postponed until section 5 .

The iso-levels with lower $\tilde{c}$ values are moving quicker compared to those with higher values in the spherical cases. This relative behaviour can be seen clearly by plotting the variation of the propagation speed in Eq. (16) across the flame brush at a given instant. This variation is shown in Figure 5a for both planar and spherical flames at $t=5 \mathrm{~ms}$. The results are shown for two combustion conditions, $u^{\prime} / s_{L}^{0}=20 \& K=1$ and $u^{\prime} / s_{L}^{0}=6 \& K=0.15$. A gradual decrease of the normalised propagation speed across the spherical flame brush is seen and this decrease is about 9 to $12 \%$ depending on the value of $u^{\prime} / s_{L}^{0}$ (larger decrease for higher $u^{\prime} / s_{L}^{0}$ ). It is to be noted that the values of the propagation speed are divided by 2 for $u^{\prime} / s_{L}^{0}=20$ cases to fit within the scale shown in Figure 5a. The statistically planar flames (open symbols) do not show any decrease across their flame brushes, except for the sharp change near the burnt side, which is for an obvious reason. The large scatter seen at $\tilde{c}=0$ is due to sharp variation of $s_{d}$ over a small range of $\tilde{c}$ near the unburnt side of the flame brush.

Typical variations of the two components, $\tilde{u} / s_{L}^{0}$ and $s_{d} / s_{L}^{0}$, across the flame brush are shown in Figure $5 \mathrm{~b}$ for the $u^{\prime} / s_{L}^{0}=6$ case at $t=5 \mathrm{~ms}$. The results for planar and spherical flames are shown respectively with dashed and solid lines and using the corresponding symbol in Figure 5a. The displacement speed is calculated using Eq. (17). The following observations can be made from this figures. (1) The normalised $\tilde{u}$ and $s_{d}$ have the same sign in the spherical case whereas they have opposite sign in the planar case. This implies that the fluid and $\tilde{c}$ iso-level move in the opposite directions in the planar flames unlike in the spherical flames. (2) The values of $s_{d} / s_{L}^{0}$ and $\tilde{u} / s_{L}^{0}$ near the leading edge of the spherical flame is much larger than in the planar flame. On the burnt side the planar flames have larger values. It is to be noted that the fluid velocity shown here is because of heat release effects since $\tilde{u}(\tilde{c}=0)$ and the velocity gradient at the burnt side are specified to be zero for the planar flames as noted earlier 
in subsection 2.3. Thus, the flow acceleration across the flame brush gives a large flow velocity on the burnt side of the planar flame brush as it is well known and this is clear in Figure 5b. In the spherical case, the burnt mixture and the unburnt mixture at large radial distance are at rest and thus the flame induced velocity has to decay to zero on both sides of the flame brush. These behaviours, especially on the unburnt side, is unclear in Figure $5 \mathrm{~b}$. Thus, the spatial variation of $\left(\tilde{u} / s_{L}^{0}\right)$ at $t=5 \mathrm{~ms}$ is shown in Figure 6, where the distance is normalised using the turbulence integral length scale, $\Lambda$. The peak flow velocity occurs near the leading edge of the spherical flame brush and it decays to zero as $r^{-2}$ in the unburnt mixture. Also, the flame brush thickness as marked roughly in Figure 6 is relatively smaller for the spherical case compared to the planar flame. The time evolution of the flame brush thickness normalised by the laminar flame thermal thickness, $\delta_{t} / \delta_{L}^{0}$, is plotted in Figure 7. The dashed lines shown in this figure represent the evolution of flame brush thickness defined using $\sqrt{c^{\prime \prime 2}}$ profile as predicted by Taylor's theory of turbulent diffusion of a passive scalar (Taylor, 1935). The solid line denotes a thickness, $\delta_{\sqrt{c^{\prime \prime 2}}}$, over which the $c$ rms value drops to $10 \%$ of its maximum value. This thickness is obtained using the computed $\widetilde{c^{\prime \prime 2}}$ and $0.4 \delta \sqrt{\sqrt{c^{\prime \prime 2}}}$ is shown in Figure 7. These results will be discussed fully in section 5. The planar flame reaches a steady value, dictated by the turbulence and thermochemical conditions, after $t^{+} \approx 2.6$, whereas there is no such steady state value for the spherical flame and its thickness keeps growing with time, which is well known in the literature. This relative behaviour is the same in other flames investigated in this study.

From the results discussed so far, it is seems that this continuous growth is because the burnt side of the flame brush is advancing slowly compared to the leading edge. This difference can be seen clearly in Figure 4 for $\widetilde{c}=0.05$ and 0.8 (also see Figure 5a). It is obvious from the discussion above that the fluid velocity at the leading edge is larger as shown in Figs. 4 to 6 and it acts together with the displacement speed in the 
spherical case. Based on these results, a simple schematic diagram can be drawn as shown in Figure 8 to represent the difference in the physical mechanisms influencing the propagation of statistically planar and spherical flames. Note the difference in directions of flow, $\widetilde{u}$, and flame displacement, $s_{d}$, speeds between the spherical and planar cases.

\subsubsection{Behaviour of $s_{d}$}

From the discussion in the previous section, it is evident that $\delta_{t}$ will influence the flame displacement speed. As noted in Eq. (17), the $s_{d}$ has two, reaction and turbulent flux, components and their typical variations across the flame brush are shown in Figure 9 for two instances, $t^{+}=4.88$ in Fig. $9 \mathrm{a}$ and 8 in Fig. $9 \mathrm{~b}$. The reaction rate contribution can be written as $\left(s_{d}^{r} / s_{L}^{0}\right)=\overline{\dot{\omega}}^{+}(1+\tau \tilde{c}) /\left|\partial \tilde{c} / \partial r^{+}\right|$. Thus, the behaviour of $\left(s_{d}^{r} / s_{L}^{0}\right)$ with $\tilde{c}$ is expected to be approximately linear according to $(1+\tau \tilde{c})$ since the variations of $\overline{\dot{\omega}}^{+}$and $\left|\partial \tilde{c} / \partial r^{+}\right|$with $\tilde{c}$ would be similar. This observation explains the variations of $\left(s_{d}^{r} / s_{L}^{0}\right)$ shown in Figure 9. The difference between the planar and spherical flames predominantly comes from $1 /\left|\partial \tilde{c} / \partial r^{+}\right|$which is related to $\delta_{t}$ shown in Figure 7 . The planar flame is thicker at $t^{+}=4.88$ and thus $\left(s_{d}^{r} / s_{L}^{0}\right)$ is larger compared to the spherical flame and the values of this displacement speed is about the same at $t^{+}=8$ because $\delta_{t}$ is nearly equal for these two flames.

The difference in the mean reaction rate variation is observed to be small in Figure 10 and this relative behaviour is also observed in other flames considered for this study. Also, the inset shows that the maximum value of the normalised mean reaction rate varies very little over the wide range of turbulence conditions of both planar and spherical flames considered in this study. The values of $\overline{\dot{\omega}}_{\max }^{+}$differ by a very small amount between the planar and spherical flames. These behaviours of $\overline{\dot{\omega}}^{+}$is observed to hold after the initial transient. The reduced sensitivity of $\overline{\dot{\omega}}^{+}$to the turbulence level 
and the flame geometry has also been reported in a direct numerical simulation study (Dunstan et al., 2012) by considering oblique and planar turbulent premixed flames established in a range of turbulence conditions.

The turbulent flux contribution, $s_{d}^{T}$, to $\left(s_{d} / s_{L}^{0}\right)$ decreases across the flame brush as shown in Figure 9, which is an expected behaviour for the planar flames. To understand its behaviour in spherical flames and for the difference seen near the leading edge, one can expand the first term of Eq. (17). This will identify an extra term of $2 \mu_{t}(\partial \tilde{c} / \partial r) /\left(r \mathrm{Sc}_{c}\right)$ in spherical flames, which will increase as $\tilde{c}$ increases in outwardly propagating flames. Since $(\partial \tilde{c} / \partial r)$ is negative for these flames, this extra term contributes negatively leading to a decrease of $s_{d}^{T}$ as $\tilde{c}$ increases. The flux contribution near the leading edge is larger in the spherical case because of the additional increase in $\partial^{2} \tilde{c} / \partial r^{2}$ resulting from the flame geometry. Thus, the difference in the $s_{d}$ of spherical and planar flames comes predominantly from the turbulent scalar flux. This is seen clearly in Figure 9, specifically at the leading edge. The influence of turbulence on the leading edge displacement speed and the consumption speed is discussed in the next subsection.

\subsubsection{Turbulent flame speed comparison}

The displacement speed of flame brush leading edge is defined as the turbulent flame speed, $s_{t}$. This quantity is of interest for theoretical investigation of turbulent flames and the influence of flame geometry on this quantity is of considerable interest for the turbulent combustion modelling (Driscoll, 2008). It has been suggested recently that $s_{t}$ is weakly sensitive to the flame geometry among freely propagating planar, strained planar and rod stablished oblique turbulent premixed flames (Dunstan et al., 2012). It must be noted that there is no "stationary value" for $s_{t}$ in the spherical flames as for the planar flames. 
The variation of $s_{t} / s_{L}^{0}$ with the turbulence Reynolds number is shown in Figures $11 \mathrm{a}$ and $12 \mathrm{a}$, and its variation with $u^{\prime} / s_{L}^{0}$ is shown as the inset. The turbulent flame speed of the spherical flames is always larger than the corresponding planar flame value for a given turbulence condition by about 10 to $20 \%$ and the higher value is due to the turbulent scalar flux contribution at the leading edge as noted in the previous subsection. The results in the inset clearly suggests an approximate relation $s_{t} \sim u^{\prime}$ when the value of the stretch factor, $K$ is kept constant. This relation was noted by Bray (1990) in his theoretical analysis using the Bray-Moss-Libby model. The increase in $s_{t}$ with $u^{\prime}$ is sharp for the high Damköhler number cases (low $K$ ) and the values of the slopes, obtained using the best linear fit, are about 6.3 and 5.1 respectively for the spherical and planar cases. These values become three times smaller for the cases with high stretch, however the relative difference in $s_{t}$ between the spherical and planar flames remains almost the same. The computed variations with $\mathrm{Re}_{t}$ shown in Figures 11a and 12a suggest a relation $s_{t} / s_{L}^{0} \simeq B \operatorname{Re}_{t}^{n}$ with $0.57 \leq n \leq 0.58$. The curves of least square fits shown in the figure for both the spherical and planar flames suggest that $B=0.20$ for the spherical and 0.18 for the planar flames. The approximate square root dependence on the turbulence Reynolds number observed here is similar to that reported by Chaudhuri et al. (2012) for the propagation speed of spherical and Bunsen flames of methane-air mixtures which are thermo-diffusively stable and do not include Darrieus-Landau instability. The approximate square root dependence observed in this study is consistent with the classical analysis of Damköhler for the thin reaction zones combustion through a hypothesis $s_{t} \sim \sqrt{D_{t} / t_{c}}$, where $D_{t}$ is the turbulent diffusivity, which is similar to $s_{L}^{0} \sim \sqrt{D / t_{c}}$, where $D$ is the molecular diffusivity, in the laminar flame theory (Peters, 1999). The results of this study suggests that the flame geometry does not impart influence on this scaling relation for turbulent flames.

Figures $11 \mathrm{~b}$ and $12 \mathrm{~b}$ show the consumption speed variation at $t^{+}=4.88$ and 8 
respectively. This speed is defined as

$$
s_{c}=\frac{1}{\rho_{u}} \int_{0}^{1} \frac{\bar{\omega}}{|\partial \tilde{c} / \partial r|} d \tilde{c} \quad=\int_{0}^{1} \frac{s_{d}^{r}}{(1+\tau \tilde{c})} d \tilde{c}
$$

The later part is obtained using $s_{d}^{r}$ defined in Eq. (17) and thus the consumption speed is the reactive component of the density weighted displacement speed integrated across the flame brush. As noted in the previous subsection, the difference between the planar and spherical flames comes predominantly through $\partial \widetilde{c} / \partial r$ and the mean reaction rate is less influenced by the flame geometry. This gives the variation of $s_{c} / s_{L}^{0}$ with $\operatorname{Re}_{t}$ similar to $s_{t} / s_{L}^{0}$, however the magnitude of $s_{c}$ is smaller than $s_{t}$ as shown in Figures 11 and 12. The difference in the consumption speeds of the planar and spherical flames at $t^{+}=8$ is very small since the flame brush thickness is nearly equal as shown in Figure 7.

\section{Discussion}

The results discussed in the previous sections suggest that the flame brush leading edge displacement speed, the turbulent flame speed, is larger for the spherical flames compared to planar flames. The propagation speed, which is the sum of fluid velocity and the displacement speed, of the leading edge grows continuously with time in the spherical flames while it reaches a nearly constant value in the planar flames. The increasing difference between the propagation speeds of the leading and trailing edges in the spherical flames yields a continuous growth of its flame brush thickness. This growth is usually attributed to turbulent diffusion in the past studies, which is different from the physical explanation given above. The aim of this section is to shed more light on these observations. 
The Kolmogorov-Petrovskii-Piskunov (KPP) analysis applied to multi-dimensional premixed flames in high Reynolds number turbulent flow gave an expression for the turbulent flame speed as (Kolla et al., 2010)

$$
s_{t}=2 \sqrt{\frac{v_{t}}{\rho_{u} S c_{c}}\left(\frac{\partial \overline{\dot{\omega}}}{\partial \widetilde{c}}\right)_{\tilde{c}=0}}+\left(\frac{v_{t}}{R S c_{c}}\right)_{\tilde{c}=0}
$$

where $v_{t}$ is the turbulent eddy viscosity and $R$ is the radius of the leading edge. Figure 10 shows that the quantity $(\partial \overline{\dot{\omega}} / \partial \widetilde{c})_{\widetilde{c}=0}$ in the planar and spherical flames are almost identical and the influence of flame brush curvature, $R^{-1}$, is responsible for the larger value of $s_{t}$ observed in figures 11 and 12 . One expects that this contribution will decrease as $R$ becomes very large and $s_{t}$ of the spherical flame to reach the planar flame value eventually. This limiting behaviour is not observed in the simulation studied here because of their finite domain size and computational time. One requires very much larger computational domain than those considered in this study, which will be addressed in future.

The Favre averaged fluid velocity at the leading edge of a turbulent spherical flame is larger than at its trailing edge as shown in Figure 8. The maximum value of this velocity will increase with time because of a continuous increase in mass burning rate resulting from the growth of the leading surface area. This increase, indeed observed in this study, results in a continuous acceleration of the leading edge of spherical flames unlike in the planar flames. Thus, a transition from turbulent deflagration to a detonation can occur eventually if the conditions are right. This transition is aggravated if the spherical flame propagates in a closed vessel under appropriate conditions.

The spatial or temporal variation of flame brush thickness has been studied in many earlier investigations and the results are summarised by Lipatnikov and Chomiak $(2002,2005)$ and an increase in the thickness with time or distance has been 
observed in these studies. Furthermore, Lipatnikov and Chomiak (2005) showed that the evolution of the measured flame brush thickness is well predicted by Taylor's theory of turbulent diffusion for a passive scalar (Taylor, 1935). This theory predicts a linear growth in $t$ for the rms displacement of a fluctuating passive scalar iso-surface when $t$ is smaller than the turbulent eddy turn over time, $t_{e}$, and this growth becomes $\sqrt{t}$ when $t$ is very much larger than $t_{e}$. Analyses using direct numerical simulation data of turbulent "V" flames offered good support for this (Minamoto et al., 2011; Dunstan et al., 2011) theory, suggesting that the turbulent diffusion plays a predominant role on the growth of the flame brush thickness. This applicability of this theory to the spherical flames studied here is tested in Figure 7, which is typical for the flames studied here. As noted earlier in section 4.2, the solid line denotes the temporal variation of $\delta \sqrt{\sqrt{c^{\prime \prime 2}}}$, a thickness over which $\sqrt{c^{\prime \prime 2}}$ drops to $10 \%$ of its maximum value. This variation is similar to $\delta_{t}$ as shown in Figure 7. The values predicted by the Taylor's theory are also shown in that figure. The gap in the theoretical curve is intentional to mark some transition from linear to square root dependence. This result suggests that the variations of $\delta_{t}$ and $\delta \sqrt{\sqrt{c^{\prime 2}}}$ does not follow the turbulent diffusion theory except for a very short initial period. The relative gap between the theoretical curve and $\delta_{t}$ increases with time. These observations suggest that the growth of $\delta_{t}$ in the spherical flame is controlled by the propagation mechanisms governed by chemical reaction, convection and turbulent diffusion. The role of turbulent diffusion for the growth of $\delta_{t}$ seems secondary compared to the convection due to the fluid velocity induced by the chemical reaction. 


\section{Conclusions}

Spherically expanding and statistically planar turbulent premixed flames of methaneair mixtures are simulated using URANS approach. The mean chemical reaction rate is modelled using the strained and unstrained flamelet models and an algebraic model of Bray (1979). The unstrained flamelet model uses $\tilde{c}$ and $\widetilde{c^{\prime \prime 2}}$ to parameterise the flamelets and the strained flamelet model (Kolla and Swaminathan, 2010) uses the mean scalar dissipation rate, $\tilde{\epsilon}_{c}$, in addition to $\tilde{c}$ and $\widetilde{c^{\prime \prime 2}}$ to parameterise the flamelets. The values of $\tilde{c}$ and $\widetilde{c^{\prime \prime 2}}$ are obtained by solving their transport equations and the mean dissipation rate is obtained using two algebraic models. These models are obtained by balancing the leading order terms of the closed transport equation for the mean scalar dissipation rate. One of this algebraic model was proposed in an earlier study (Kolla et al., 2009) for statistically planar flames and the second model includes the effects of mean curvature. The turbulence is modelled using the $k-\varepsilon$ equations.

These models are first validated by computing a spherical methane-air flame investigated experimentally in an earlier study (Hainsworth, 1985). A good comparison between the computed and measured flame ball growth rate is observed for the strained flamelet model and the other two combustion submodels yield a faster growth.

Statistically planar and spherical flames, fourteen flames in total, experiencing low and high turbulence stretch rates are computed using strained flamelet model and these flames are analysed in detail to understand the influence of geometry on their propagation. For the conditions investigated in this study, including curvature corrections in the algebraic model for $\tilde{\epsilon}_{c}$ did not influence the flame propagation. Detailed analyses of the computed flames showed that the advancement of the leading edge is aided by the local fluid velocity in the spherical case. In the planar flames, the directions for the fluid flow and the advancing leading edge are opposite. The planar flame showed 
a steady propagation once a balance between the local flow and displacement speeds is achieved for a given turbulence conditions. The spherical flames accelerated continuously because of the compounded effects of flow and leading edge displacement. This continuous acceleration cause the heat release induced induced convective effects to be dominant for the growth of the flame brush thickness.

The flame geometry is observed to influence the magnitude of turbulent scalar flux at the leading edge, spherical flames showing larger magnitude compared to the planar flames for a given turbulence and thermo-chemical conditions. The mean reaction rate is found to be less influenced by the flame geometry. Thus, the influence of flame geometry on the turbulent flame speed, leading edge displacement speed, is observed to result from the contribution of the turbulence scalar flux. The turbulent flame speed, $s_{t}$, of the spherical flames is observed to be 10 to $20 \%$ higher than the corresponding planar flame values for the conditions investigated in this study. For a constant value of turbulence stretch rate, $s_{t} \sim u^{\prime}$ as noted by Bray (1990) and this scaling is observed for both planar and spherical flames. The values of $s_{t}$, normalised by the laminar flame speed, for the fourteen flames computed in this study scales as $\operatorname{Re}_{t}^{n}$ with $0.57 \leq$ $n \leq 0.58$. This scaling is consistent with the classical analysis of Damköhler. The consumption speed also shows a similar scaling with $\mathrm{Re}_{t}$. The results presented in this work encourages the use of strained flamelet model to simulate turbulent combustion in modern spark-ignition engines, which involve expanding flame balls.

\section{Acknowledgment}

We like to acknowledge Professor Cheng at MIT for providing a copy of the MS thesis containing the experimental data used in this study. 


\section{References}

Abdel-Gayed, R. G., Al-Khishali, K. J., and Bradley, D. 1984. Turbulent burning velocities and flame straining in explosions. Proc. R. Soc. Lond. A, 391 (1801), 393414.

Abdel-Gayed, R. G., Bradley, D., and Lawes, M. 1987. Turbulent burning velocities: a general correlation in terms of straining rates. Proc. R. Soc. Lond. A, 414 (1847), 389-413.

Abdel-Gayed, R. G., Bradley, D., Lawes, M., and Lung, F.K-K. 1988. Premixed turbulent burning during explosions. Proc. Combust. Inst., 21, 497-504.

Albin, E., and D'Angelo, Y. 2012. Assessment of the evolution equation modelling approach for three-dimensional expanding wrinkled premixed flames. Combust. Flame, 159, 1932-1948.

Andrews, G. E., Bradley, D., and Lwakabamba, S. B. 1975. Measurement of turbulent burning velocity for large turbulent reynolds numbers. Proc. Combust. Inst., 15, $655-664$.

Ashurst, W. T. 1995. A simple illustration of turbulent flame ball growth. Combust. Sci. Technol., 104, 19-32.

Ashurst, W. T., Checkel, M. D., and Ting, D. S. K. 1994. The eddy structure model of turbulent flamelet propagation, the expanding spherical and steady planar cases. Combust. Sci. Technol., 99, 51-74.

Baum, M., and Poinsot, T. 1995. Effects of mean flow on premixed flame ignition. Combust. Sci. Technol., 106, 19-39. 
Beretta, G. P., Rashidi, M., and Keck, J. C. 1983. Turbulent flame propagation and combustion in spark ignition engines. Combust. Flame, 52, 217-245.

Blizard, N. C., and Keck, J. C. 1974. Experimental and theoretical investigation of turbulent burning model for internal combustion engines. Tech. Rep. 740191. SAE International, Warrendale, PA, USA.

Bradley, D., Gaskell, P. H., and Gu, X. J. 1996. Burning velocities, Markstein lengths, and flame quenching for spherical methane-air flames: A computational study. Combust. Flame, 104, 176-198.

Bradley, D., Haq, M. Z., Hicks, R. A., Kitagawa, T., Lawes, M., Sheppard, C. G. W., and Woolley, R. 2003. Turbulent burning velocity, burned gas distribution, and associated flame surface definition. Combust. Flame, 133, 415-430.

Bradley, D., Lawes, M., and Mansour, M. S. 2009. Explosion bomb measurements of ethanol-air laminar gaseous flame characteristics at pressures up to $1.4 \mathrm{MPa}$. Combust. Flame, 156, 1462-1470.

Bradley, D., Lawes, M., and Mansour, M. S. 2011. Correlation of turbulent burning velocities of ethanolair, measured in a fan-stirred bomb up to 1.2 MPa. Combust. Flame, 158, 123-138.

Bradley, D., Lawes, M., Scott, M. J., and Mushi, E. M. J. 1994. Afterburning in spherical premixed turbulent explosions. Combust. Flame, 99, 581-590.

Bradley, D., Sheppard, C. G. W., Woolley, R., Greenhalgh, D. A., and Lockett, R. D. 2000. The development and structure of flame instabilities and cellularity at low markstein numbers in explosions. Combust. Flame, 122, 195-209. 
Bray, K. N. C. 1979. The interaction between turbulence and combustion. Proc. Combust. Inst., 17, 223-233.

Bray, K. N. C. 1990. Studies of the burning velocity. Proc. R. Soc. Lond. A, 431, 315335.

Bray, K. N. C., Libby, P. A., and Moss, J. B. 1985. Unified modeling approach for premixed turbulent combustion. 1. general formulation. Combust. Flame, 61, 87102.

Chakraborty, N., Champion, N., Mura, A., and Swaminathan, N. 2011. 2.3 Scalardissipation-rate approach, Turbulent Premixed Flames. In Swaminathan, N., and Bray, K. N. C., (Eds.). Cambridge University Press, Cambridge, UK. pp. 74-102.

Chakraborty, N., Rogerson, J. W., and Swaminathan, N. 2010. The scalar gradient alignment statistics of flame kernels and its modelling implications for turbulent premixed combustion. Flow Turbul. Combust., 85, 25-55.

Chaudhuri, S., Wu, F., Zhu, D., and Law, C. K. 2012. Flame speed and self-similar propagation of expanding turbulent premixed flames. Phys. Rev. Let., 108 (4), 044503-1-5.

Checkel, M. D., and Thomas, A. 1994. Turbulent combustion of premixed flames in closed vessels. Combust. Flame, 96, 351-370.

Colin, O., and Truffin, K. 2011. A spark ignition model for large eddy simulation based on an FSD transport equation (ISSIM-LES). Proc. Combust. Inst., 33, 3097-3104.

Corvellec, C., Bruel, P., and Sabel'nikov, V. A. 1999. A time-accurate scheme for the calculations of unsteady reactive flows at low Mach number. Int. J. Numer. Meth. $F l ., 29(2), 207227$. 
Corvellec, C., Bruel, P., and SabelNikov, V. A. 2000. Turbulent premixed flames in the flamelet regime: burning velocity spectral properties in the presence of countergradient diffusion. Combust. Flame, 120 (4), 585-588.

Driscoll, J. F. 2008. Turbulent premixed combustion: Flamelet structure and its effect on turbulent burning velocities. Prog. Energy Combust. Sci., 34, 91-134.

Dunstan, T. D., Swaminathan, N., and Bray, K. N. C. 2012. Influence of flame geometry on turbulent premixed flame propagation: a DNS investigation. J. Fluid Mech., 709, 191-222.

Dunstan, T. D., Swaminathan, N., Bray, K. N. C., and Cant, R. S. 2011. Geometrical properties and turbulent flame speed measurements in stationary premixed v-flames using direct numerical simulation. Flow Turbulence Combust., 709, 237-259.

Frank, J. H., Kalt, P. A. M., and Bilger, R. W. 1999. Measurements of conditional velocities in turbulent premixed flames by simultaneous OH PLIF and PIV. Combust. Flame, 116 (1-2).

Fureby, C. 2005. A fractal flame-wrinkling large eddy simulation model for premixed turbulent combustion. Proc. Combust. Inst., 30, 593-601.

Groff, E. G. 1987. An experimental evaluation of an entrainment flame-propagation model. Combust. Flame, 67, 153-162.

Hainsworth, E. 1985. Study of free turbulent premixed flames. Master's thesis, Mass. Inst. Technol., Cambridge, MA, USA.

Haq, M. Z., Sheppard, C. G. W., Woolley, R., Greenhalgh, D. A., and Lockett, R. D. 2002. Wrinkling and curvature of laminar and turbulent premixed flames. Combust. Flame, 131, 1-15. 
Jenkins, K. W., and Cant, R. S. 2002. Curvature effects on flame kernels in a turbulent environment. Proc. Combust. Inst., 29, 2023-2029.

Jenkins, K. W., Klein, M., Chakraborty, N., and Cant, R. S. 2006. Effects of strain rate and curvature on the propagation of a spherical flame kernel in the thin-reactionzones regime. Combust. Flame, 145, 415-434.

Jones, W. P. 1994. Turbulence modelling and numerical solution methods for variable density and combustion flows, Turbulent Reacting Flows. In Libby, P. A., and Williams, F. A., (Eds.). Academic Press, London, UK. pp. 309-374.

Kalt, P. A. M., Frank, J. H., and Bilger, R. W. 1998. Laser imaging of conditional velocities in premixed propane-air flames by simulataneous OH PLIF and PIV.Proc. Combust. Inst., 27 (1), 751.

Kaminski, C. F., Hult, J., Aldén, M., Lindenmaier, S., Dreizler, A., Maas, U., and Baum, M. 2000. Spark ignition of turbulent methane/air mixtures revealed by timeresolved planar laser-induced fluorescence and direct numerical simulations. Proc. Combust. Inst., 28, 399-405.

Kee, R. J., Grcar, J. F., Smooke, M. D., and Miller, J. A. 1985. A fortran program for modeling steady laminar one-dimensional premixed flames. Tech. Rep. SAND858240. Sandia National Laboratories, Livermore, CA, USA.

Klein, M., Chakraborty, N., and Cant, R. S. 2008. Effects of turbulence on selfsustained combustion in premixed flame kernels: a direct numerical simulation (DNS) study. Flow Turbul. Combust., 81, 583-607.

Klein, M., Chakraborty, N., Jenkins, K. W., and Cant, R. S. 2006. Effects of initial radius on the propagation of premixed flame kernels in a turbulent environment. Phys. of Fluids, 18 (5), 055102. 
Kolla, H., Rogerson, J. W., Chakraborty, N., and Swaminathan, N. 2009. Scalar dissipation rate modeling and its validation. Combust. Sci. Technol., 181, 518-535.

Kolla, H., Rogerson, J. W., and Swaminathan, N. 2010. Validation of a turbulent flame speed model across combustion regimes. Combust. Sci. Technol., 182, 284-308.

Kolla, H., and Swaminathan, N. 2010. Strained flamelets for turbulent premixed flames, I: Formulation and planar flame results. Combust. Flame, 157, 943-954.

Kolla, H., and Swaminathan, N. 2011. Influence of turbulent scalar mixing physics on premixed flame propagation. J. Combust., 2011, 1-8.

Law, C. K., and Sung, C. J. 2000. Structure, aerodynamics, and geometry of premixed flamelets. Prog. Energy Combust. Sci., 26, 459-505.

Lawes, M., Ormsby, M. P., Sheppard, C. G. W., and Woolley, R. 2012. The turbulent burning velocity of iso-octane/air mixtures. Combust. Flame, 159, 1949-1959.

Lecocq, G., Richard, S., Colin, O., and Vervisch, L. 2011. Hybrid presumed pdf and flame surface density approaches for large-eddy simulation of premixed turbulent combustion. part 2: Early flame development after sparking. Combust. Flame, 158, $1215-1226$.

Libby, P. A. 1985. Theory of normal premixed turbulent flames revisited. Prog. Energy Combust. Sci., 11, 83-96.

Lipatnikov, A. N., and Chomiak, J. 2000. Transient and geometrical effects in expanding turbulent flames. Combust. Sci. Technol., 154, 75-117.

Lipatnikov, A. N., and Chomiak, J. 2002. Turbulent flame speed and thickness: phenomenology, evaluation, and application in multi-dimensional simulations .Prog. Energy Combust. Sci., 28, 1-74. 
Lipatnikov, A. N., and Chomiak, J. 2004. Application of the Markstein number concept to curved turbulent flames. Combust. Sci. Technol., 176, 331-358.

Lipatnikov, A. N., and Chomiak, J. 2005. Molecular transport effects on turbulent flame propagation and structure. Prog. Energy Combust. Sci., 31, 1-73.

Lutz, A. E., Kee, R. J., Grcar, J. F., and Rupley, F. M. 1997. OPPDIF: A fortran program for computing opposed-flow diffusion flames. Tech. Rep. SAND96-8243. Sandia National Laboratories, Livermore, CA, USA.

Minamoto, Y., Fukushima, N., Tanahashi, M., Miyauchi, T., Dunstan, T. D., and Swaminathan, N. 2011. Effect of flow-geometry on turbulence-scalar interaction in premixed flames. Phys. Fluids, 23, 125107.

Nwagwe, I. K., Weller, H. G., Tabor, G. R., Gosman, A. D., Lawes, M., Sheppard, C. G. W., and Wooley, R. 2000. Measurements and large eddy simulations of turbulent premixed flame kernel growth. Proc. Combust. Inst., 28, 59-65.

van Oijen, J. A., Groot, G. R. A., Bastiaans, R. J. M., and de Goey, L. P. H. 2005. A flamelet analysis of the burning velocity of premixed turbulent expanding flames. Proc. Combust. Inst., 30, 657-664.

Patankar, S. V. 1980. Numerical heat transfer and fluid flow. Taylor and Francis Group, London, UK.

Peters, N. 1999. The turbulent burning velocity for large-scale and small-scale turbulence. J. Fluid Mech., 384, 107-132.

Poinsot, T., Candel, S., and Trouvé, A. 1995. Applications of direct numerical simulation to premixed turbulent combustion. Prog. Energy Combust. Sci., 21, 531-576. 
Poinsot, T., and Veynante, D. 2005. Theoretical and Numerical Combustion, 2nd edn. R.T. Edwards, Inc., Philadelphia, PA, USA.

Pope, S. B. 1987. Turbulent premixed flames. Ann. Rev. Fluid Mech, 19, 237-270.

Pope, S. B., and Cheng, W. K. 1986. Statistical calculations of spherical turbulent flames. Proc. Combust. Inst., 21, 1473-1481.

Renou, B., Boukhalfa, A., Puechberty, D., and Trinité, M. 2000. Local scalar flame properties of freely propagating premixed turbulent flames at various Lewis numbers. Combust. Flame, 123, 507-521.

Renou, B., Mura, A., Samson, E., and Boukhalfa, A. 2002. Characterization of the local flame structure and the flame surface density for freely propagating premixed flames at various Lewis numbers. Combust. Sci. Technol., 174, 143-179.

Schmid, H.-P., Habisreuther, P., and Leuckel, W. 1998. A model for calculating heat release in premixed turbulent flames. Combust. Flame, 113, 79-91.

Smith, G. P., Golden, D. M., Frenklach, M., Moriarty, N. W., Eiteneer, B., Goldenberg, M., Bowman, C. T., Hanson, R. K., Song, S., Gardiner Jr., W. C, Lissianski, V. V., and Qin, Z. 2000. [Online] Available from: http://www.me.berkeley.edu/gri_mech/.

Swaminathan, N., Bilger, R. W., and Ruetsch, G. R. 1997. Interdependence of the instantaneous flame front structure and the overall scalar flux in turbulent premixed flames. Combust. Sci. and Technol., 128 (1), 73.

Swaminathan, N., and Bray, K. N. C. 2005. Effect of dilatation on scalar dissipation in turbulent premixed flames. Combust. Flame, 143, 549-565. 
Swaminathan, N., and Bray, K. N. C. 2011. Fundamentals and Challenges, Turbulent Premixed Flames. In Swaminathan, N., and Bray, K. N. C., (Eds.). Cambridge University Press, Cambridge, UK. pp. 1-40.

Tabaczynski, R. J., Trinker, F. H., and Shannon, B. A. S. 1980. Further refinement and validation of a turbulent flame propagation model for spark-ignition engines. Combust. Flame, 39, 111-121.

Tabor, G., and Weller, H. G. 2004. Large eddy simulation of premixed turbulent combustion using flame surface wrinkling model. Flow Turbul. Combust., 72, 1-27.

Taylor, G. I. 1935. Statistical theory of turbulence IV. Diffusion in a turbulent air stream. Proc. R. Soc. London, Ser. A, 151, 151-478.

Thévenin, D. 2005. Three-dimensional direct simulations and structure of expanding turbulent methane flames. Proc. Combust. Inst., 30, 629-637.

Thévenin, D., Gicquel, O., De Charentenay, J., Hilbert, R., and Veynante, D. 2002. Two- versus three-dimensional direct simulations of turbulent methane flame kernels using realistic chemistry. Proc. Combust. Inst., 29, 2031-2039.

Veynante, D., Trouv, A., Bray, K. N. C., and Mantel, T. 1997. Gradient and countergradient scalar transport in turbulent premixed flames. J. Fluid Mech., 332, 263-293.

Zhang, S.W., and Rutland, C.J. 1995. Premixed flame effects on turbulence and pressure-related terms. Combust. Flame, 102, 447-461. 


\section{List of Figures}

1 Regime diagram of turbulent premixed combustion (Peters, 1999) with the flame conditions considered for this study. The filled symbols correspond to two flame stretch parameters: $K=0.15(\bullet)$ and $K=1.0(\mathbf{\theta})$ and the open triangle $(\triangle)$ is for the experimental case of Hainsworth

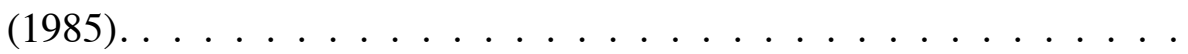

2 Comparison of numerical and experimental (Hainsworth, 1985) results: (a) radius as a function of time and (b) flame propagation speed as a function of radius. All quantities are normalised as indicated. . .

3 Spatial variation of $\tilde{c}$ at five different times, 0 to $8 \mathrm{~ms}$ at an interval of $2 \mathrm{~ms}$, in (a) planar and (b) spherical flames having $u^{\prime} / s_{L}^{0}=6$ and $K=0.15$. Dashed lines show the variation at $t=0 . \ldots \ldots$

4 Temporal variation of propagation speed of $\tilde{c}$ iso-levels in planar and spherical turbulent flames for (a) $u^{\prime} / s_{L}^{0}=6$ and $K=0.15$, and (b) $u^{\prime} / s_{L}^{0}=18$ and $K=1$. Results are shown for three different iso-levels.

5 The variation of propagation speed across the flame brush is shown in (a) for planar (open symbols) and spherical (closed) flames with $u^{\prime} / s_{L}^{0}=6$ and $K=0.15$ (circle), and $u^{\prime} / s_{L}^{0}=20$ and $K=1$ (square) at $t^{+}=4.88(t=5 \mathrm{~ms})$. The two components, $s_{d}$ (symboled lines) and $\tilde{u}$ (lines), are shown in (b) for the $u^{\prime} / s_{L}^{0}=6$ case. . . . . . . . .

6 Spatial variation of $\tilde{u} / s_{L}^{0}$ at $t^{+}=4.88$ for the cases with $u^{\prime} / s_{L}^{0}=6$ and $K=0.15$. The locations of $\tilde{c}=0.05$ and 0.95 in the respective flames are marked to indicate the flame brush sizes. . . . . . . . . . . . . 48

$7 \quad$ Evolution of flame brush thickness with time in flames with $u^{\prime} / s_{L}^{0}=18$ and $K=1 \ldots \ldots \ldots \ldots \ldots \ldots \ldots \ldots \ldots \ldots$ 
8 Schematic diagrams showing the propagation mechanism in a statistically planar and spherical flames. Dashed and solid arrows represent the flow and iso-level displacement directions respectively. . . . . . .

9 Reaction rate and turbulent flux contributions to $\left(s_{d} / s_{L}^{0}\right)$ for both planar and spherical flames with $u^{\prime} / s_{L}^{0}=6$ and $K=0.15$ at: (a) $t^{*}=1.14$ $\left(t^{+}=4.88\right)$ and $(\mathrm{b}) t^{*}=1.87\left(t^{+}=8\right) \ldots \ldots \ldots \ldots$

10 Variation of normalised mean reaction rate across the flame brush for both planar and spherical flames, with $u^{\prime} / s_{L}^{0}=6$ and $K=0.15$ at (a) $t^{+}=4.88$ and (b) $t^{+}=8$, and the inset shows the variation of $\overline{\dot{\omega}}_{\max }^{+}$with $\left(u^{\prime} / s_{L}^{0}\right)$ for the various flames considered in this study, where $\overline{\dot{\omega}}^{+}=\overline{\dot{\omega}} \delta_{L}^{0} /\left(\rho_{u} s_{L}^{0}\right) \ldots \ldots \ldots \ldots \ldots \ldots \ldots \ldots$

11 Planar (open symbols) and spherical (closed) flame speeds for the cases simulated in this study are plotted against the turbulence Reynolds number. The inset shows the variation with $u^{\prime} / s_{L}^{0}$. All flame speeds are taken at $t^{+}=5.86(t=6 \mathrm{~ms})$ in (a) normalised turbulent flame speed and (b) normalised consumption speed. The symbols correspond to those shown in Figure $1 . \ldots \ldots 52$

12 Planar (open symbols) and spherical (closed) flame speeds for the cases simulated in this study are plotted against the turbulence Reynolds number. The inset shows the variation with $u^{\prime} / s_{L}^{0}$. All flame speeds are taken at $t^{+}=8(t=8.2 \mathrm{~ms})$ in (a) normalised turbulent flame speed and (b) normalised consumption speed. The symbols correspond to those shown in Figure $1 . \ldots \ldots$. . . . . . . . . . . 53 


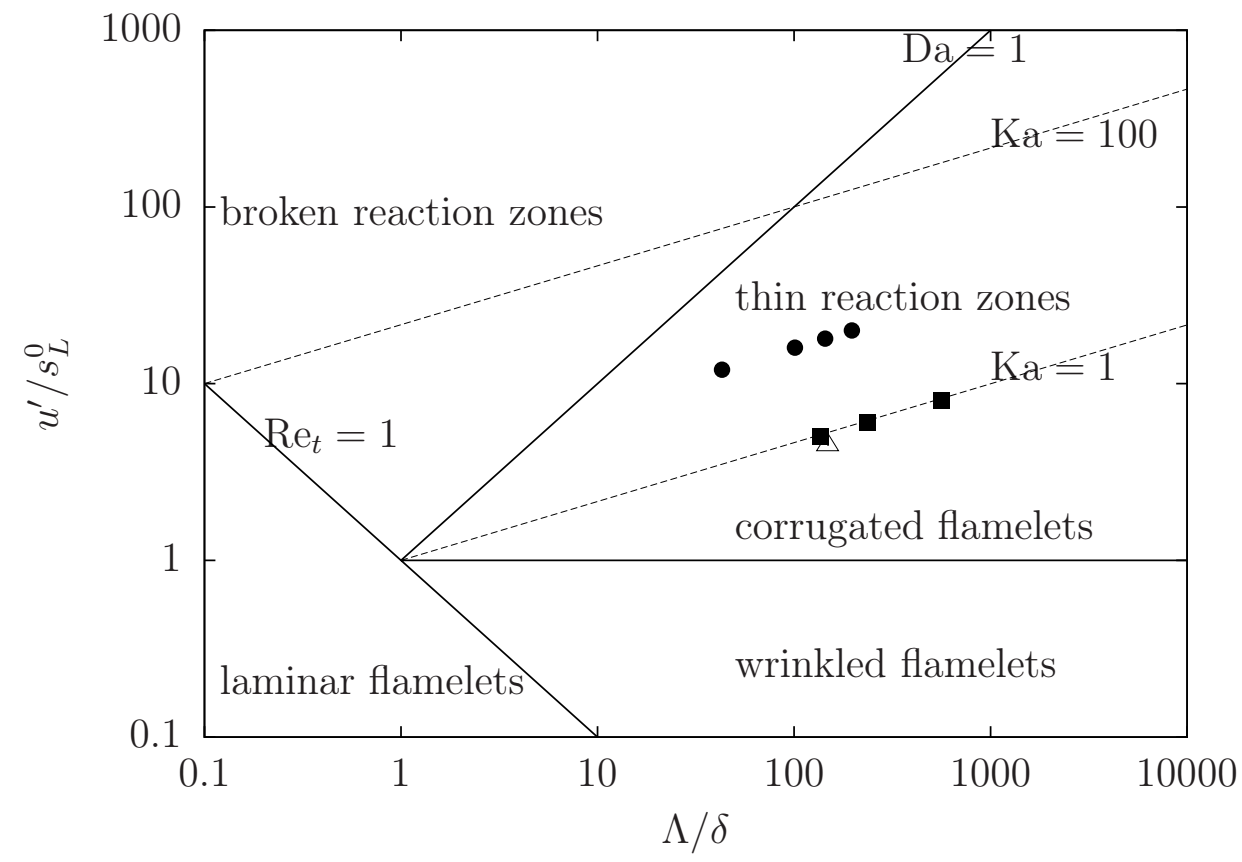

Figure 1: Regime diagram of turbulent premixed combustion (Peters, 1999) with the flame conditions considered for this study. The filled symbols correspond to two flame stretch parameters: $K=0.15(\bullet)$ and $K=1.0(\boldsymbol{\square})$ and the open triangle $(\triangle)$ is for the experimental case of Hainsworth (1985). 

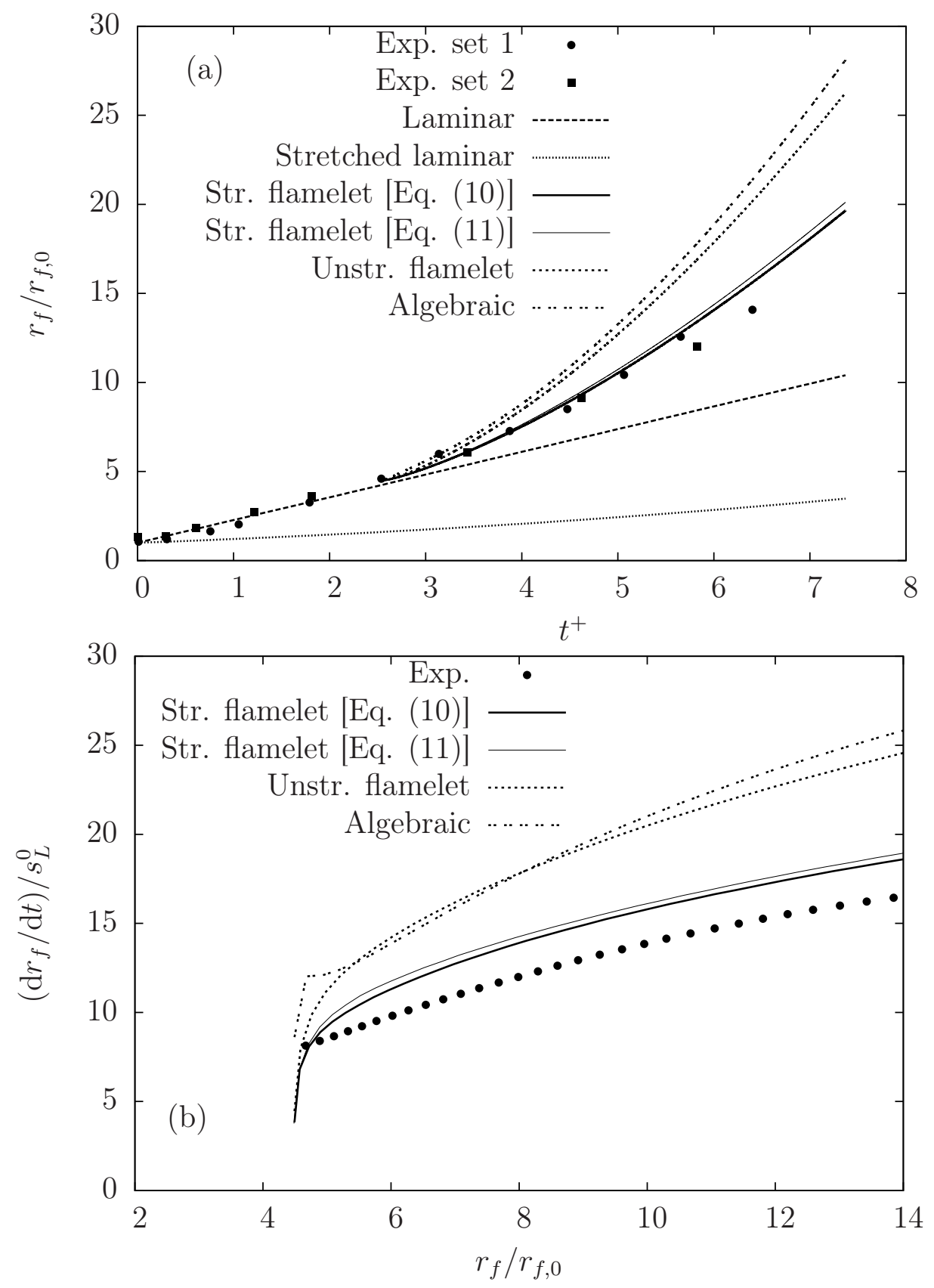

Figure 2: Comparison of numerical and experimental (Hainsworth, 1985) results: (a) radius as a function of time and (b) flame propagation speed as a function of radius. All quantities are normalised as indicated. 

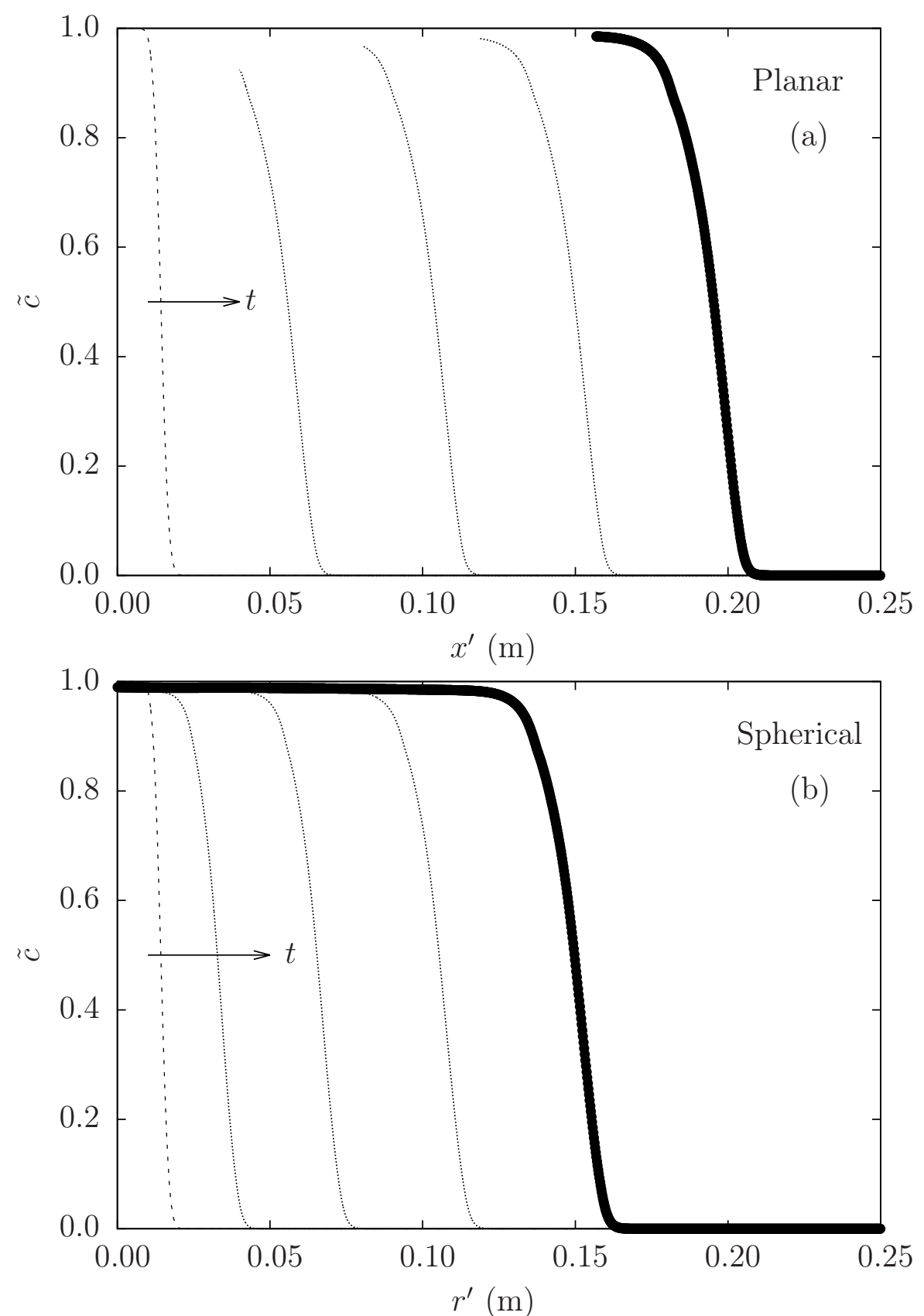

Figure 3: Spatial variation of $\tilde{c}$ at five different times, 0 to $8 \mathrm{~ms}$ at an interval of $2 \mathrm{~ms}$, in (a) planar and (b) spherical flames having $u^{\prime} / s_{L}^{0}=6$ and $K=0.15$. Dashed lines show the variation at $t=0$. 

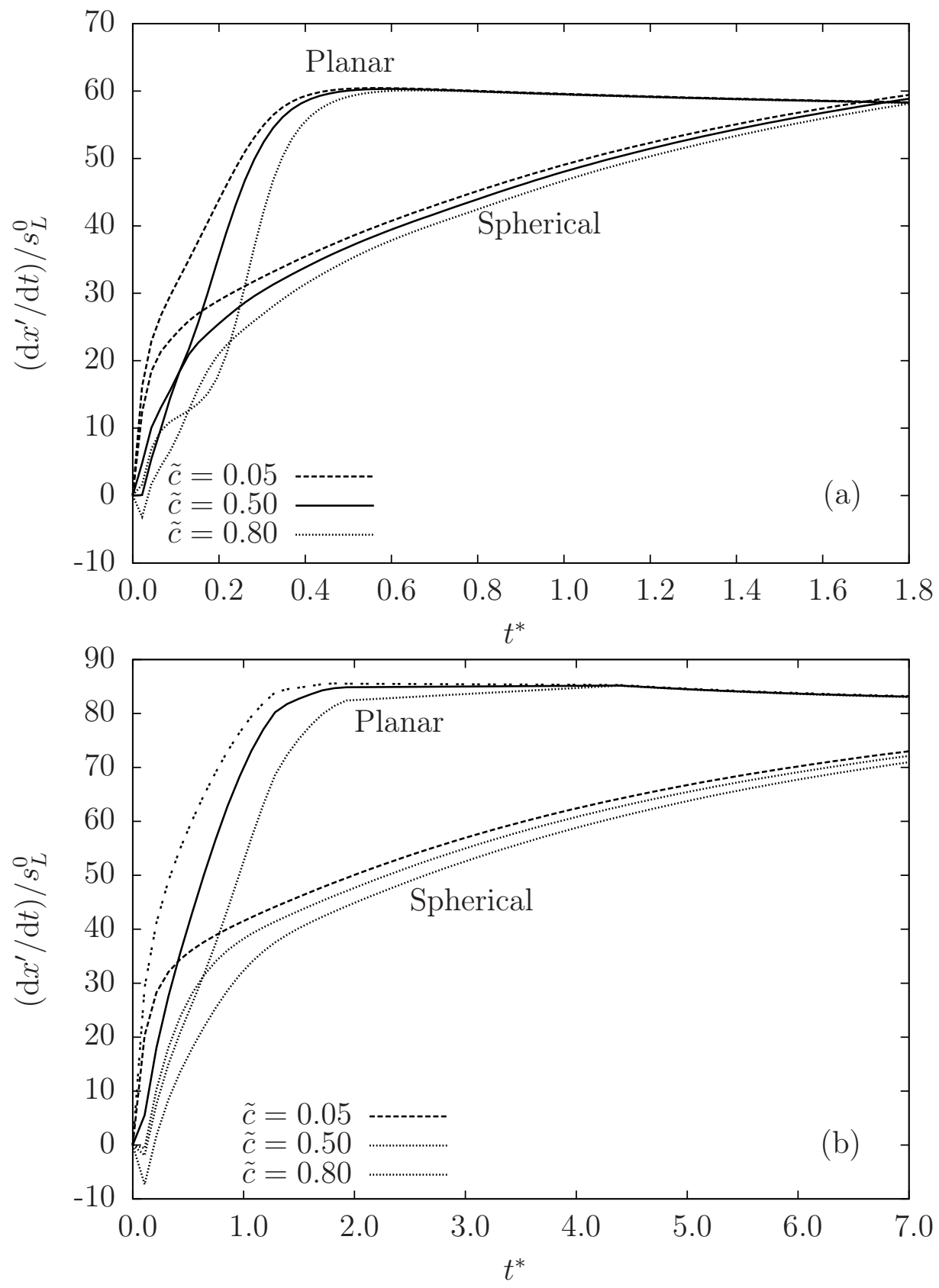

Figure 4: Temporal variation of propagation speed of $\tilde{c}$ iso-levels in planar and spherical turbulent flames for (a) $u^{\prime} / s_{L}^{0}=6$ and $K=0.15$, and (b) $u^{\prime} / s_{L}^{0}=18$ and $K=1$. Results are shown for three different iso-levels. 

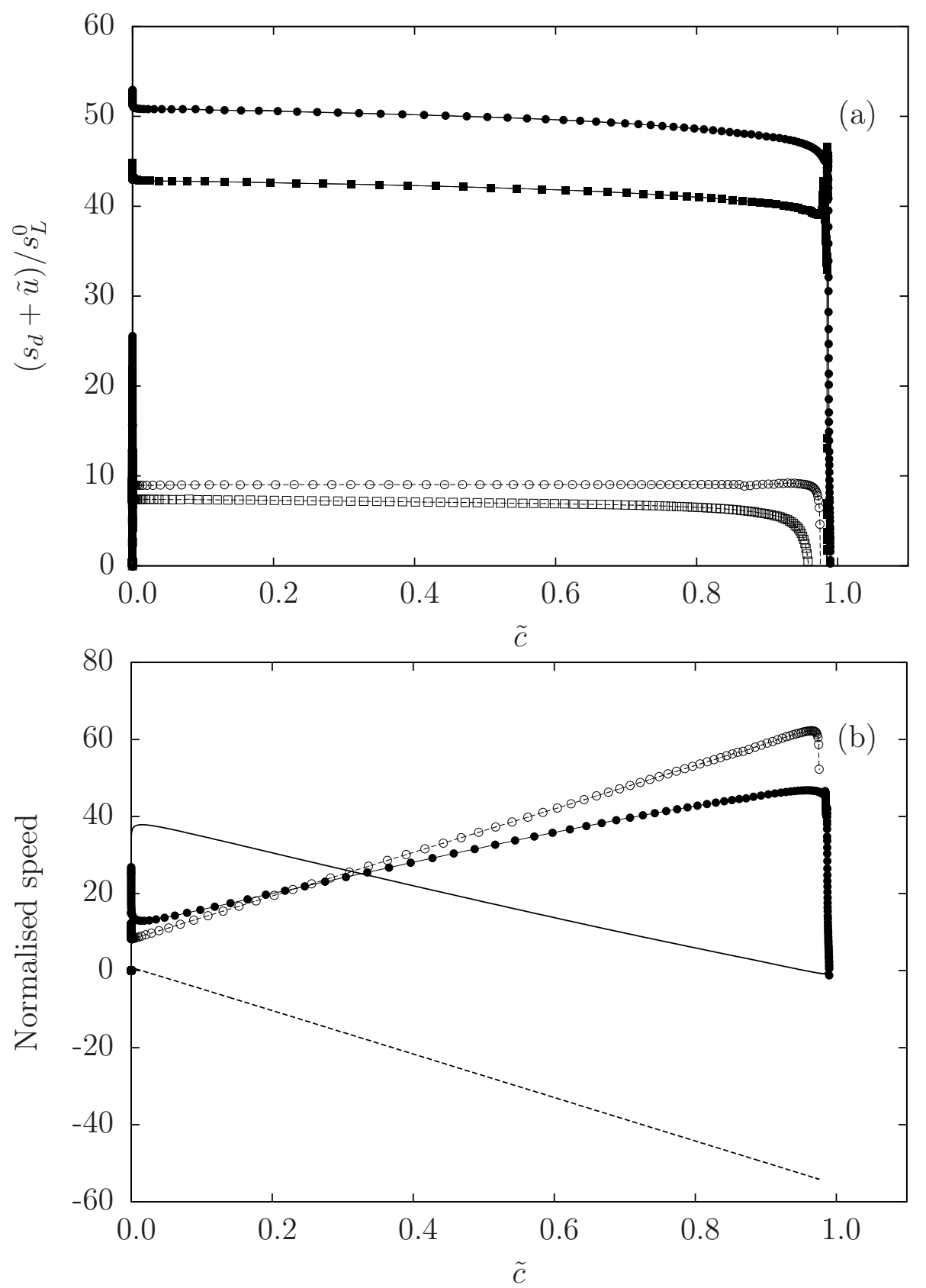

Figure 5: The variation of propagation speed across the flame brush is shown in (a) for planar (open symbols) and spherical (closed) flames with $u^{\prime} / s_{L}^{0}=6$ and $K=0.15$ (circle), and $u^{\prime} / s_{L}^{0}=20$ and $K=1$ (square) at $t^{+}=4.88(t=5 \mathrm{~ms})$. The two components, $s_{d}$ (symboled lines) and $\tilde{u}$ (lines), are shown in (b) for the $u^{\prime} / s_{L}^{0}=6$ case. 


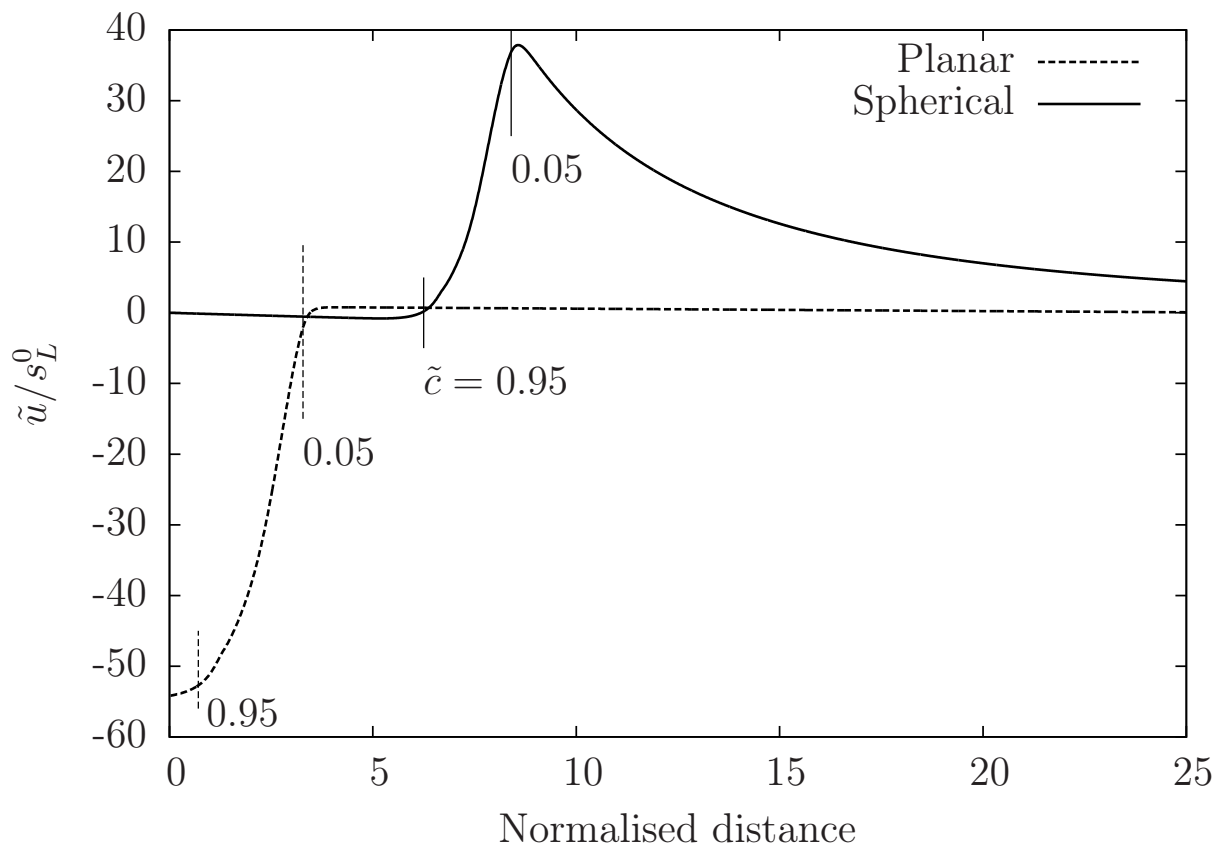

Figure 6: Spatial variation of $\tilde{u} / s_{L}^{0}$ at $t^{+}=4.88$ for the cases with $u^{\prime} / s_{L}^{0}=6$ and $K=0.15$. The locations of $\tilde{c}=0.05$ and 0.95 in the respective flames are marked to indicate the flame brush sizes.

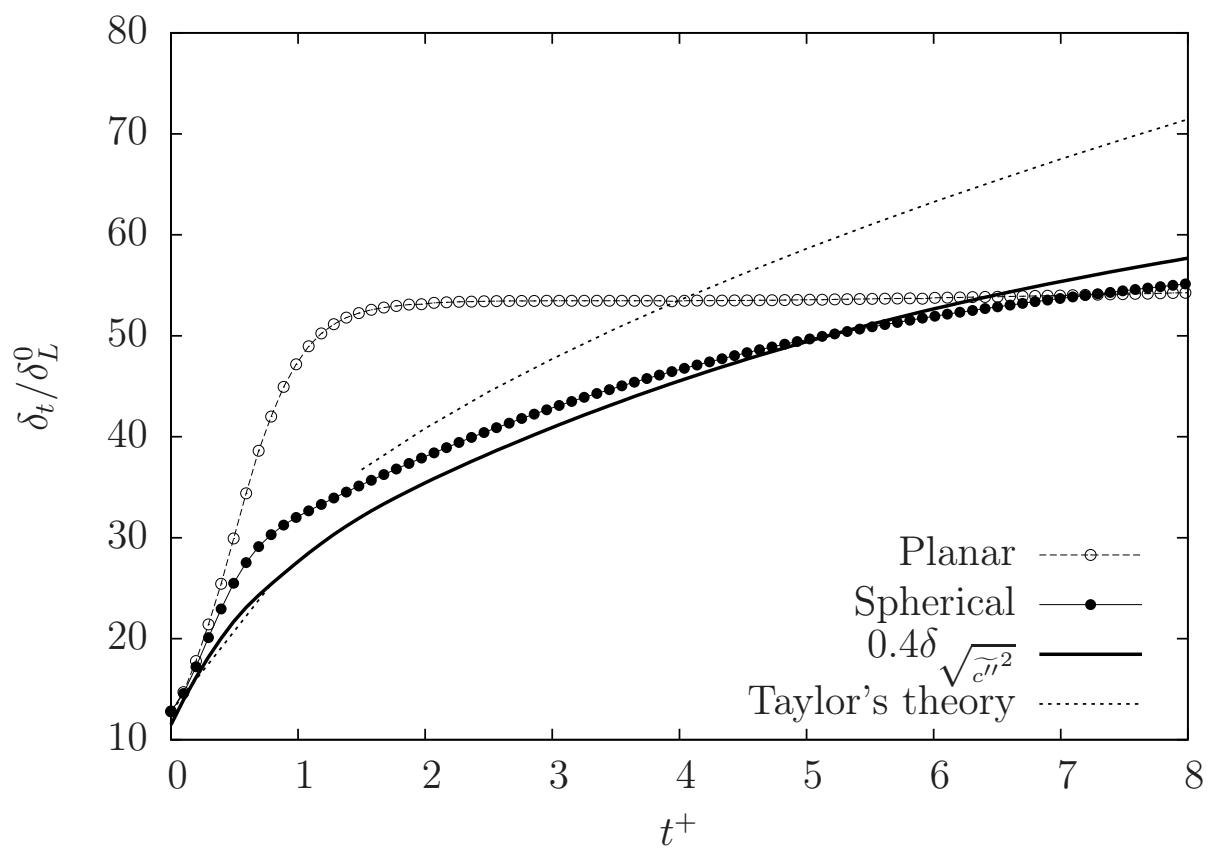

Figure 7: Evolution of flame brush thickness with time in flames with $u^{\prime} / s_{L}^{0}=18$ and $K=1$. 


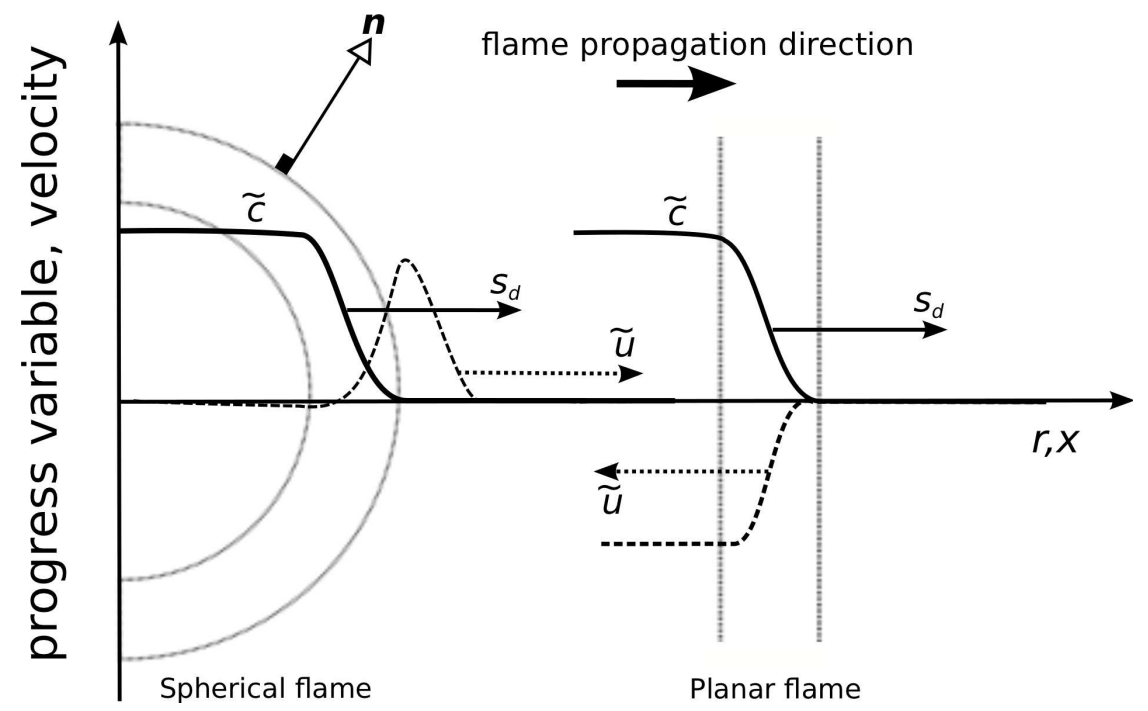

Figure 8: Schematic diagrams showing the propagation mechanism in a statistically planar and spherical flames. Dashed and solid arrows represent the flow and iso-level displacement directions respectively. 

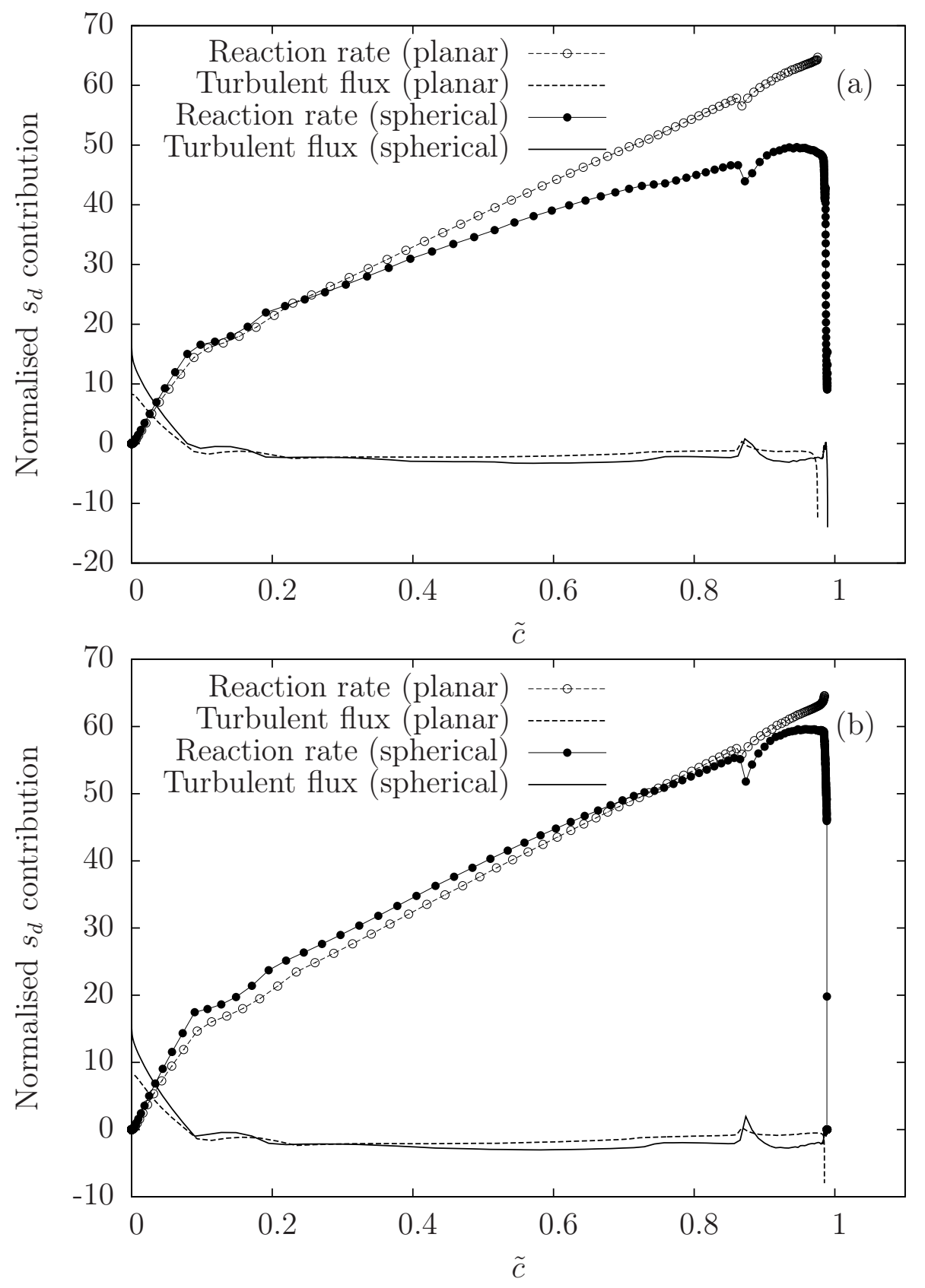

Figure 9: Reaction rate and turbulent flux contributions to $\left(s_{d} / s_{L}^{0}\right)$ for both planar and spherical flames with $u^{\prime} / s_{L}^{0}=6$ and $K=0.15$ at: (a) $t^{*}=1.14\left(t^{+}=4.88\right)$ and (b) $t^{*}=1.87\left(t^{+}=8\right)$. 

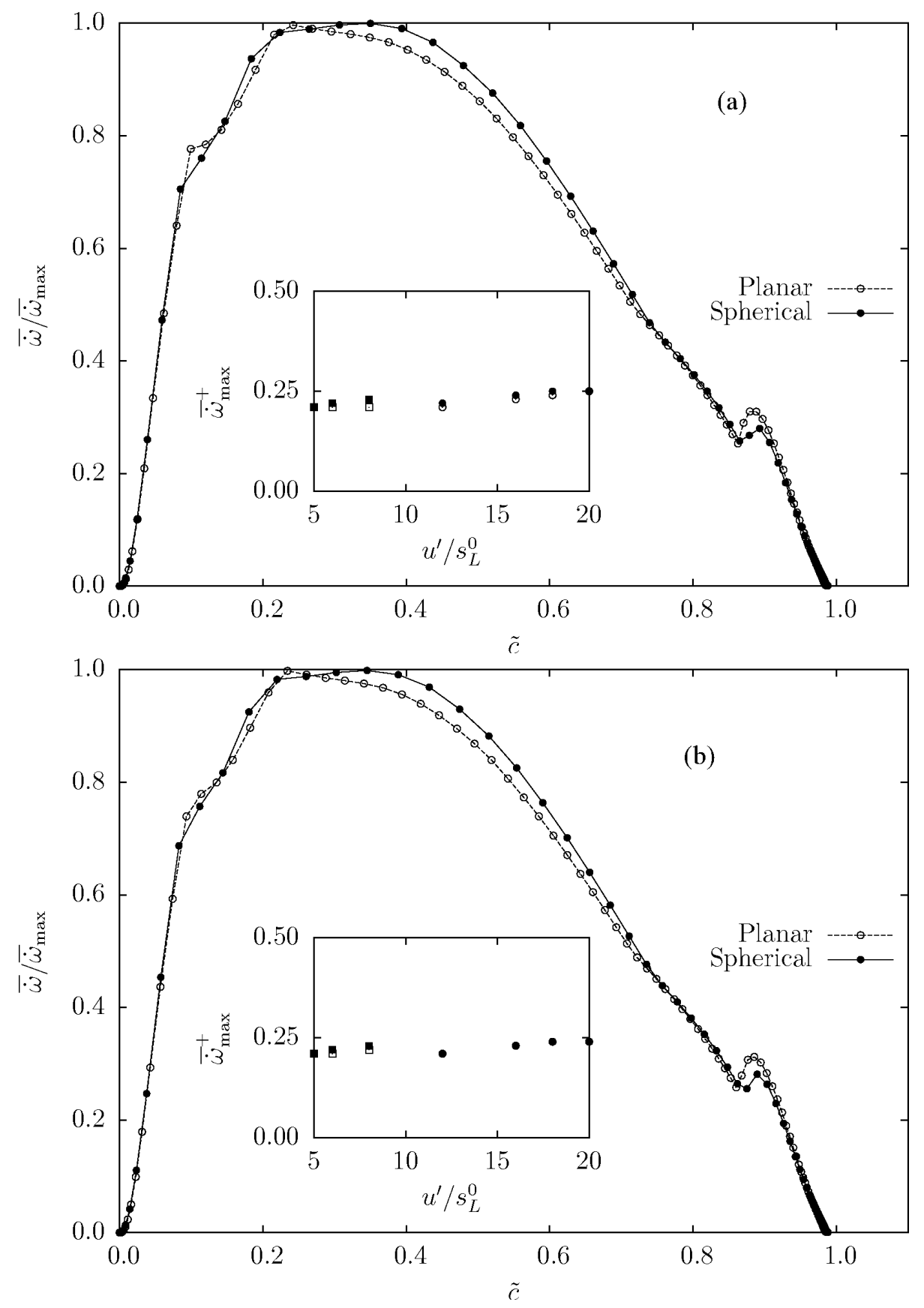

Figure 10: Variation of normalised mean reaction rate across the flame brush for both planar and spherical flames, with $u^{\prime} / s_{L}^{0}=6$ and $K=0.15$ at (a) $t^{+}=4.88$ and (b) $t^{+}=8$, and the inset shows the variation of $\overline{\dot{\omega}}_{\max }^{+}$with $\left(u^{\prime} / s_{L}^{0}\right)$ for the various flames considered in this study, where $\overline{\dot{\omega}}^{+}=\overline{\dot{\omega}} \delta_{L}^{0} /\left(\rho_{u} s_{L}^{0}\right)$. 

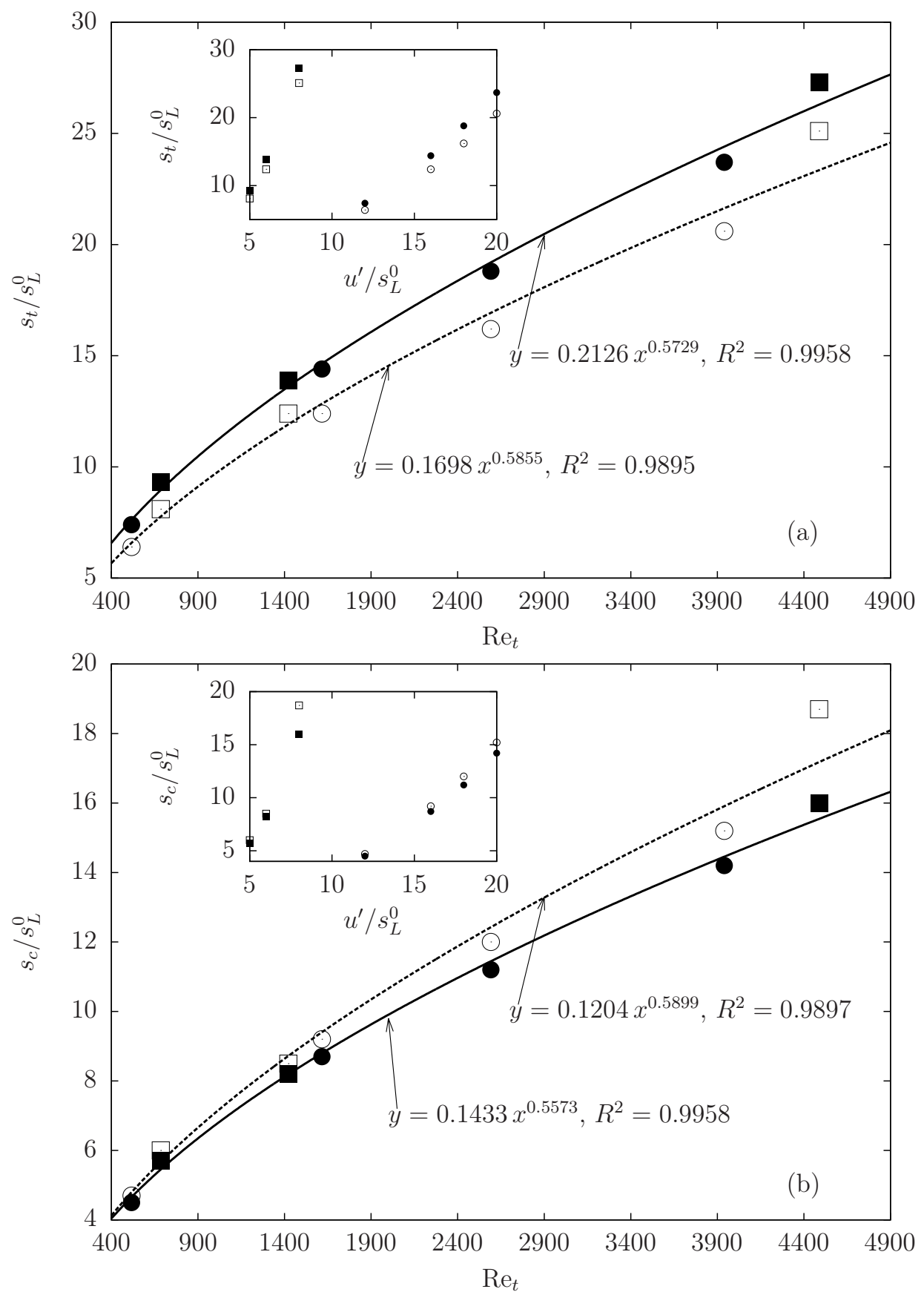

Figure 11: Planar (open symbols) and spherical (closed) flame speeds for the cases simulated in this study are plotted against the turbulence Reynolds number. The inset shows the variation with $u^{\prime} / s_{L}^{0}$. All flame speeds are taken at $t^{+}=5.86(t=6 \mathrm{~ms})$ in (a) normalised turbulent flame speed and (b) normalised consumption speed. The symbols correspond to those shown in Figure 1. 

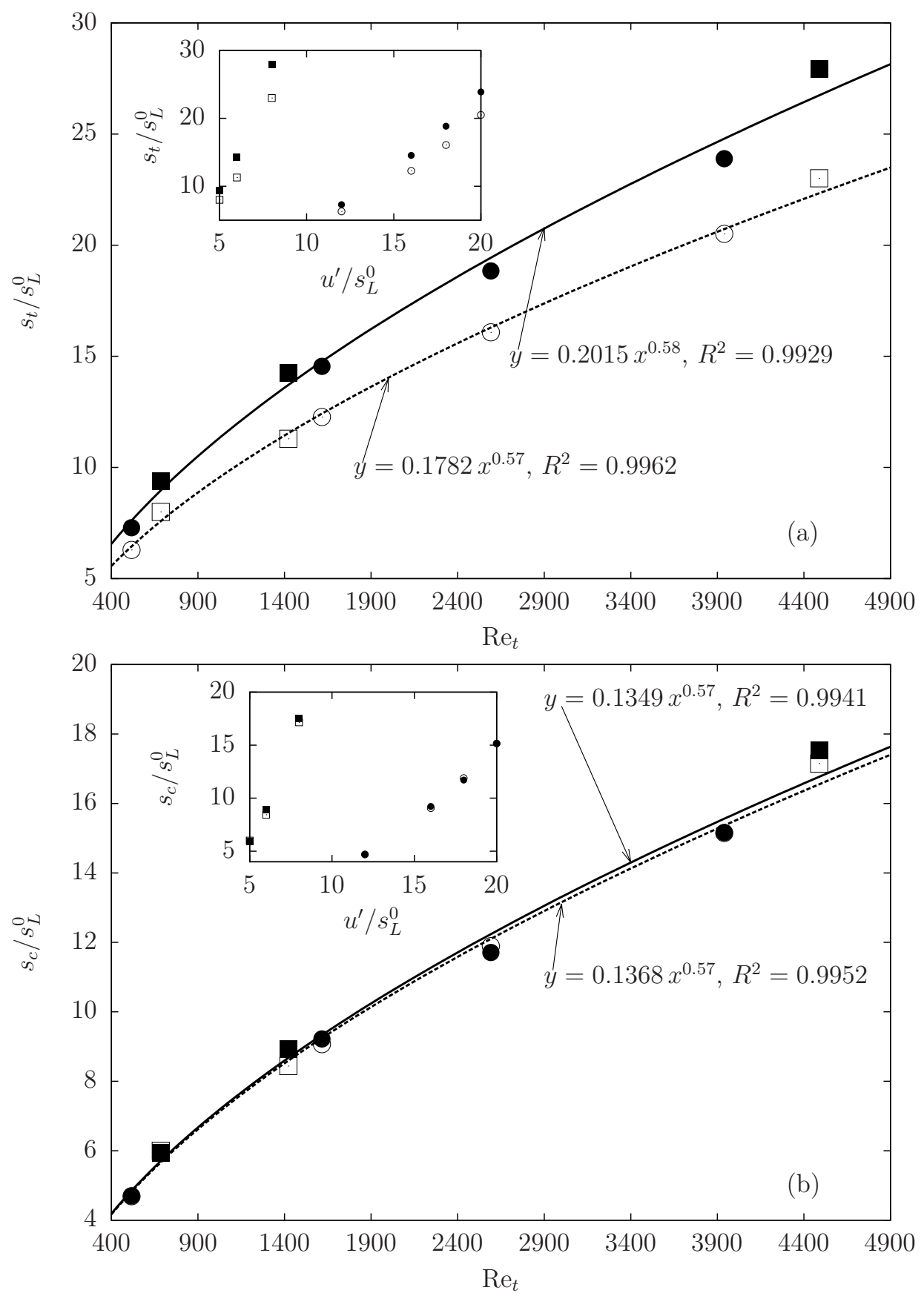

Figure 12: Planar (open symbols) and spherical (closed) flame speeds for the cases simulated in this study are plotted against the turbulence Reynolds number. The inset shows the variation with $u^{\prime} / s_{L}^{0}$. All flame speeds are taken at $t^{+}=8(t=8.2 \mathrm{~ms})$ in (a) normalised turbulent flame speed and (b) normalised consumption speed. The symbols correspond to those shown in Figure 1. 